Discrete Comput Geom 35:551-595 (2006)

DOI: $10.1007 / \mathrm{s} 00454-006-1234-2$

\title{
Volume Computation for Polytopes and Partition Functions for Classical Root Systems
}

\author{
M. Welleda Baldoni, ${ }^{1}$ Matthias Beck, ${ }^{2}$ Charles Cochet, ${ }^{3}$ and Michèle Vergne ${ }^{4}$ \\ ${ }^{1}$ Departimento di Matematica, University of Rome Tor Vergata, \\ via della Ricerca Scientifica, 00133 Roma, Italy \\ baldoni@mat.uniroma2.it \\ ${ }^{2}$ Department of Mathematics, San Francisco State University, \\ San Francisco, CA 94312, USA \\ beck@math.sfsu.edu \\ ${ }^{3}$ U.F.R. de Mathematiques, case 7012, Université Paris 7, \\ 2 place Jussieu, 75251 Paris Cedex 05, France \\ cochet@math.jussieu.fr \\ ${ }^{4}$ Centre de Mathematiques, Ecole Polytechnique, \\ 91128 Palaiseau Cedex, France \\ vergne@math.polytechnique.fr
}

\begin{abstract}
This paper presents an algorithm to compute the value of the inverse Laplace transforms of rational functions with poles on arrangements of hyperplanes. As an application, we present an efficient computation of the partition function for classical root systems.
\end{abstract}

\section{Introduction}

The ultimate goal of this work is to present an algorithm for a fast computation of the partition function of classical root systems. We achieve this goal in somewhat more general terms, namely we develop algorithms to compute the volume of a polytope and its discrete analog, the number of integer points in the polytope. These formulas, in turn, are inverse Laplace transforms of certain rational functions, and our work can be viewed in these general terms.

Let $U$ be a finite-dimensional real vector space of dimension $r$. Denote its dual vector space $U^{*}$ by $V$. Consider a set of elements

$$
\mathcal{A}=\left\{\alpha_{1}, \alpha_{2}, \ldots, \alpha_{N}\right\}
$$


of non-zero vectors of $V$. We assume that the convex cone $\mathcal{C}(\mathcal{A})$ generated by nonnegative linear combinations of the elements $\alpha_{i}$ is an acute convex cone in $V$ with non-empty interior.

The elements $\ell$ in $V$ produce linear functions $u \mapsto \ell(u)$ on the complexified vector space $U_{\mathbb{C}}$. In particular, to the set $\mathcal{A}$ we associate the arrangement of hyperplanes

$$
\mathcal{H}_{\mathbb{C}}(\mathcal{A}):=\bigcup_{i=1}^{N}\left\{u \in U_{\mathbb{C}} \mid \alpha_{i}(u)=0\right\}
$$

in $U_{\mathbb{C}}$ and its complement

$$
U_{\mathbb{C}}(\mathcal{A}):=\left\{u \in U_{\mathbb{C}} \mid \prod_{i=1}^{N} \alpha_{i}(u) \neq 0\right\} .
$$

We denote by $\mathcal{R}_{\mathcal{A}}$ the ring of rational functions on $U_{\mathbb{C}}(\mathcal{A})$ with poles along $\mathcal{H}_{\mathbb{C}}(\mathcal{A})$. Then each element $\varphi \in \mathcal{R}_{\mathcal{A}}$ can be written as $P / Q$ where $P$ is a polynomial function on $r$ complex variables and $Q$ is a product of elements, not necessarily distinct, of $\mathcal{A}$.

Our first aim is to present an algorithm to compute the value of the inverse Laplace transform of functions in $\mathcal{R}_{\mathcal{A}}$ at a point $h \in V$. In other words, we study the value at a point $h \in V$ of convolutions of a number of Heaviside distributions $\varphi \mapsto \int_{0}^{\infty} \varphi\left(t \alpha_{i}\right) d t$. The first theoretical ingredient is the notion of Jeffrey-Kirwan residues [17]. Going a step further, De Concini and Procesi [13] proved that one can compute Jeffrey-Kirwan residues using maximal nested sets (in short MNSs), a combinatorial tool related to no-broken-circuit bases of the set of vectors $\mathcal{A}$.

The applications in view are volume computation for polytopes, enumeration of integral points in polytopes, and, more generally, discrete or continuous integration of polynomial functions over polytopes. Indeed, Szenes and Vergne [22], refining a formula of Brion and Vergne [7], stated formulas for the volume and number of integral points in polytopes involving Jeffrey-Kirwan residues.

Consider the polytope

$$
\Pi_{\mathcal{A}}(h):=\left\{x \in \mathbb{R}^{N} \mid \sum_{i=1}^{N} x_{i} \alpha_{i}=h, x_{i} \geq 0\right\} .
$$

As a function of $h$, the volume of $\Pi_{\mathcal{A}}(h)$ is a piecewise-defined polynomial. The chambers of polynomiality in the parameter space $V$ are polyhedral cones.

Our programs are extremely efficient for computing the volume of the polytope $\Pi_{\mathcal{A}}(h)$ when $\mathcal{A}$ is a classical root system. An important fact is that our algorithm can work with formal parameters, thus giving the polynomial volume formula for $\Pi_{\mathcal{A}}(h)$ when $h$ runs over a particular chamber.

For an analogous theory for integral-point enumeration, we have to assume that the $\alpha_{i}$ are vectors in a lattice $V_{\mathbb{Z}}$. For $h \in V_{\mathbb{Z}}$, the function $N_{\mathcal{A}}(h)$ which associates to the vector $h$ the number of integral points in $\Pi_{\mathcal{A}}(h)$, that is the number of ways to represents the vector $h$ as a sum of a certain number of vectors $\alpha_{i}$, is called the (vector-)partition function of $\mathcal{A}$. For example, for $B_{2}$, given a vector $\left(h_{1}, h_{2}\right)$ with integral coordinates we would like to compute the number $N_{B_{2}}(h)$ of vectors $\left(x_{i}\right) \in \mathbb{Z}_{+}^{4}$ such that

$$
x_{1}\left(\begin{array}{l}
1 \\
0
\end{array}\right)+x_{2}\left(\begin{array}{l}
0 \\
1
\end{array}\right)+x_{3}\left(\begin{array}{r}
1 \\
-1
\end{array}\right)+x_{4}\left(\begin{array}{l}
1 \\
1
\end{array}\right)=\left(\begin{array}{l}
h_{1} \\
h_{2}
\end{array}\right) .
$$


As a function of $h$, the number $N_{\mathcal{A}}(h)$ of integral points in $\Pi_{\mathcal{A}}(h)$ is a piecewisedefined quasipolynomial, and again the chambers of quasipolynomiality are polyhedra in $V$ [12], [20], [21].

In this paper we describe an efficient algorithm for MNS computation for classical root systems. This algorithm for MNS gives rise to programs for the Kostant partition function for the classical root systems $A_{n}, B_{n}, C_{n}$, and $D_{n}$. Again, our algorithm works with a formal parameter $h$ that is assumed to be confined to a particular chamber.

These calculations are valuable because partition functions play a fundamental role also in representation theory of semisimple Lie algebras $\mathfrak{g}$. Indeed, partition functions arise naturally when we want to compute the multiplicity of a weight in a finitedimensional representation or the tensor-product decomposition of two representations, both being basic problems to understand characters of representations. Cochet [9], [10] has obtained very efficient algorithms for both these problems implementing results of this paper.

We conclude by describing the way the paper is organized. Section 2 introduces Laplace transforms and polytopes. In Section 3 we recall the Jeffrey-Kirwan residue and its link with counting formulas. De Concini-Procesi's MNSs are described in Section 4, as well as how they are related to Jeffrey-Kirwan residues. Section 5 describes our general algorithm for MNS computations. Details of particular cases of the algorithm for the root systems $A_{n}, B_{n}, C_{n}$ and $D_{n}$ are examined in Sections 7-10. Finally, comparative tests of our programs with existing softwares are performed in Section 11.

A number of theoretical results related to the function $N_{\mathcal{A}}(h)$ when $\mathcal{A}$ is a subset of the system $A_{n}$ can be found in [1] (as, for example, the computation of the volume of the Chan-Robbins polytope).

Computer programs for volume computation/integral-point enumeration in polytopes have only been implemented in the very recent past, most notably Latt $\mathrm{E}$ [14], [15] and barvinok [6], both of which are implementations of Barvinok's algorithm [3]. To the best of our knowledge, these two are the only general programs for volume computation/integral-point enumeration in polytopes. More specialized programs include algorithms of Baldoni et al. for flow polytopes [2] and Beck and Pixton for the Birkhoff polytope [4].

Our programs have been especially designed for classical root systems, are faster than all actual existing softwares, and can compute new examples that were not reachable by previous algorithms. Note in particular that our programs can perform computations for $N_{\mathcal{A}}(h)$ for $\mathcal{A}_{n}$ at least up to $n=10$ (11 coordinates vector). For $\mathcal{B}_{n}, \mathcal{C}_{n}$, and $\mathcal{D}_{n}$ the algorithms are efficient at least up to $n=6$. For our methods (as well as for LattE), the size of the vector $h$ has little affect on the computation time. Recall that our methods can also calculate the multivariate quasipolynomials $h \mapsto N_{\mathcal{A}}(h)$ when $h$ varies on a chamber, and as a particular case for a fixed $h$ the function $k \mapsto N_{\mathcal{A}}(k h)$ which is the Ehrhart quasipolynomial in $k$.

\section{Laplace Transform and Polytopes}

We start by briefly recalling the notations of the Introduction, aiming to relate the inverse of the Laplace transform with various counting formulas for a polytope. A good introduction on this theme is the survey article [24]. 


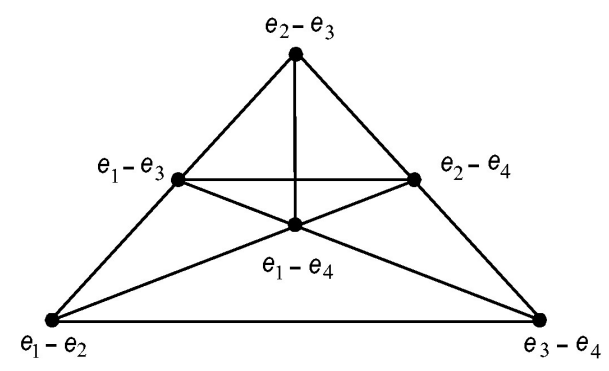

Fig. 1. The seven chambers for $A_{3}$.

\subsection{Laplace Transform}

Let $U$ be a finite-dimensional real vector space of dimension $r$ with dual space $V$. We fix the choice of a Lebesgue measure $d h$ on $V$. Consider a set

$$
\mathcal{A}=\left\{\alpha_{1}, \alpha_{2}, \ldots, \alpha_{n}\right\}
$$

of non-zero vectors of $V$. We assume that the set of vectors $\alpha_{i}$ spans $V$. For any subset $S$ of $V$, we denote by $\mathcal{C}(S)$ the convex cone generated by non-negative linear combinations of elements of $S$. We assume that the convex cone $\mathcal{C}(\mathcal{A})$ is acute in $V$ with non-empty interior.

Let $\mathcal{V}_{\text {sing }}(\mathcal{A})$ be the union of the boundaries of the cones $\mathcal{C}(S)$, where $S$ ranges over all the subsets of $\mathcal{A}$. The complement of $\mathcal{V}_{\text {sing }}(\mathcal{A})$ in $\mathcal{C}(\mathcal{A})$ is by definition the open set $\mathcal{C}_{\text {reg }}(\mathcal{A})$ of regular elements. A connected component $\mathfrak{c}$ of $\mathcal{C}_{\text {reg }}(\mathcal{A})$ is called a chamber of $\mathcal{C}(\mathcal{A})$. Figures 1 and 2 represent slices of the cones $\mathcal{C}\left(A_{3}\right)$ and $\mathcal{C}\left(B_{3}\right)$, where the dots represent the intersection of a slice with a ray $\mathbb{R}_{\geq 0} \alpha_{i}$ hence showing the chambers. Note that the chambers for $B_{r}$ and $C_{r}$ are the same (as roots in $B_{r}$ and $C_{r}$ are proportional). In dimension 3, the root system $A_{3}$ is isomorphic to $D_{3}$. See [2] for the computation of chambers. Very little is known about the total number of chambers. On the other hand, given a vector $h$, it is easy to compute the equations of the chamber containing $h$. This

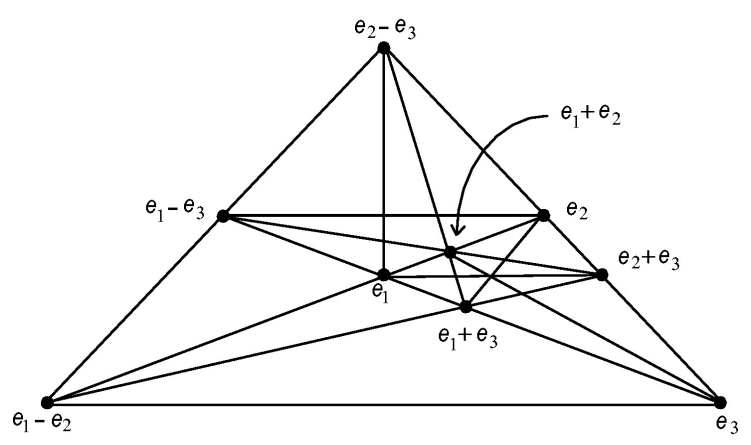

Fig. 2. The 23 chambers for $B_{3}$. 
Table 1. Number of chambers and computation time.

\begin{tabular}{ccccccc}
\hline & $\mathrm{A}$ & $\mathrm{B}$ & $\mathrm{C}$ & $\mathrm{D}$ & $\mathrm{F}$ & $\mathrm{G}$ \\
\hline 1 & 1 & 1 & 1 & & & \\
& $(0 \mathrm{~s})$ & $(0 \mathrm{~s})$ & $(0 \mathrm{~s})$ & & & 5 \\
2 & 2 & 3 & 3 & 1 & & $(0 \mathrm{~s})$ \\
& $(0 \mathrm{~s})$ & $(0 \mathrm{~s})$ & $(0 \mathrm{~s})$ & $(0 \mathrm{~s})$ & & \\
3 & 7 & 23 & 23 & 7 & & \\
& $(1 \mathrm{~s})$ & $(8 \mathrm{~s})$ & $(8 \mathrm{~s})$ & $(1 \mathrm{~s})$ & & \\
4 & 48 & 695 & 695 & 133 & 12,946 & \\
& $(23 \mathrm{~s})$ & $(11 \mathrm{~min})$ & $(11 \mathrm{~min})$ & $(90 \mathrm{~s})$ & $(3 \mathrm{~d} 16 \mathrm{~h})$ & \\
5 & 820 & $>26,905$ & $>26,905$ & 12,926 & & \\
& $(19 \mathrm{~min})$ & $?$ & $?$ & $(1 \mathrm{~d} 5 \mathrm{~h})$ & & \\
6 & 44,288 & $?$ & $?$ & $?$ & & \\
& $(24 \mathrm{~d} 18 \mathrm{~h})$ & & & & & \\
\hline
\end{tabular}

was done in [2] and [11]. We have incorporated this small part of the corresponding program in our programs for classical root systems.

Table 1 represents the only numbers of chambers that have been computed (and the computation time).

Consider now a cone $\mathcal{C}(S)$ spanned by a subset $S$ of $\mathcal{A}$ and let $p$ be a function on $\mathcal{C}(S)$. We assume that $p$ is the restriction to $\mathcal{C}(S)$ of a polynomial function on $V$. By superposing such functions $p$, we obtain a space $\mathcal{L P}(V, \mathcal{A})$ of locally polynomial functions on $\mathcal{C}(\mathcal{A})$. For $f \in \mathcal{L P}(V, \mathcal{A})$, the restriction of $f$ to any chamber $\mathfrak{c}$ of $\mathcal{C}(\mathcal{A})$ is given by a polynomial function.

The Laplace transform $L(f)$ of such a function $f$ is defined as follows. Consider the dual cone $\mathcal{C}(\mathcal{A})^{*} \subset U$ of $\mathcal{C}(\mathcal{A})$ defined by

$$
\mathcal{C}(\mathcal{A})^{*}=\{u \in U \mid\langle h, u\rangle \geq 0 \text { for all } h \in \mathcal{C}(\mathcal{A})\} .
$$

Then, for $u$ in the interior of the cone $\mathcal{C}(\mathcal{A})^{*}$, the integral

$$
L(f)(u)=\int_{\mathcal{C}(\mathcal{A})} e^{-\langle h, u\rangle} f(h) d h
$$

is convergent. It is easy to see that the function $L(f)$ is the restriction to $\mathcal{C}(\mathcal{A})^{*}$ of a function in $\mathcal{R}_{\mathcal{A}}$. (Recall that $\mathcal{R}_{\mathcal{A}}$ is the ring of rational functions $P / Q$ on $U$ where $P$ is a polynomial function on $U$ and $Q$ is a product of elements of $\mathcal{A}$.) It is easy [7] to characterize the functions $L(f)$ on $U$ arising this way.

Let $v$ be a subset of $\{1,2, \ldots, n\}$. We say that $v$ is generating (respectively basic) if the set $\left\{\alpha_{i} \mid i \in v\right\}$ generates (respectively is a basis of) the vector space $V$.

Every basic subset is of cardinality $r$ and we write $\operatorname{Bases}(\mathcal{A})$ for the set of basic subsets. Given $\sigma \in \operatorname{Bases}(\mathcal{A})$, the associated basic fraction is

$$
f_{\sigma}=\frac{1}{\prod_{i \in \sigma} \alpha_{i}} .
$$

In a system of coordinates (depending on $\sigma$ ) on $U$ where $\alpha_{i}(u)=u_{i}$ (for $i \in \sigma$ ), such a 
basic fraction is simply of the form

$$
\frac{1}{u_{1} u_{2} \cdots u_{r}} \text {. }
$$

Define $\mathcal{G}(U, \mathcal{A}) \subset \mathcal{R}_{\mathcal{A}}$ as the linear span of functions $1 / \prod_{i \in v} \alpha_{i}^{n_{i}}$, where $v$ is generating and $n_{i}$ are positive integers. The following proposition gives the characterization we were speaking of and is easy to prove:

Proposition 2.1 [7]. If $f$ is a locally polynomial function on $\mathcal{C}(\mathcal{A})$, the Laplace transform $L(f)$ of $f$ is the restriction to $\mathcal{C}(\mathcal{A})^{*}$ of a function in $\mathcal{G}(U, \mathcal{A})$. Reciprocally, for any generating set $v$ and every set of positive integers $n_{i}>0$, there exists a locally polynomial function $f$ on $V$ such that

$$
\frac{1}{\prod_{i \in \nu} \alpha_{i}(u)^{n_{i}}}=\int_{\mathcal{C}(\mathcal{A})} e^{-\langle h, u\rangle} f(h) d h
$$

for any $u$ in the interior of $\mathcal{C}(\mathcal{A})^{*}$.

We define the inverse Laplace transform $L^{-1}: \mathcal{G}(U, \mathcal{A}) \rightarrow \mathcal{L} \mathcal{P}(V, \mathcal{A})$ as follows. For $\varphi \in \mathcal{G}(U, \mathcal{A})$, the function $L^{-1} \varphi$ is the unique locally polynomial function that satisfies

$$
\varphi(u)=\int_{\mathcal{C}(\mathcal{A})} e^{-\langle h, u\rangle}\left(L^{-1} \varphi\right)(h) d h
$$

for any $u \in \mathcal{C}(\mathcal{A})^{*}$.

In the next sections we explain the relation between Laplace transforms and the enumeration of integral points of families of polytopes. We will see in Section 4 that one can write efficient formulas for the inversion of Laplace transforms in terms of residues, whose algorithmic implementation is working in a quite impressive way, at least for low dimension.

\subsection{Volume and Number of Integral Points of a Polytope}

In this subsection we consider a sequence

$$
\mathcal{A}^{+}=\left[\alpha_{1}, \alpha_{2}, \ldots, \alpha_{N}\right]
$$

of non-zero elements of $\mathcal{A}$. We assume that each element $\alpha \in \mathcal{A}$ occurs in the sequence; in particular $N \geq n$ and the set $\mathcal{A}^{+}$spans $V$.

Remark 2.2. In all our examples the sequence $\mathcal{A}^{+}$will not have multiplicities, so that we will freely identify $\mathcal{A}^{+}$and $\mathcal{A}$.

We now introduce the notion of a partition polytope.

We consider the space $\mathbb{R}^{N}$ with its standard basis $\omega_{i}$ and Lebesgue measure $d x$. If $x=\sum_{i=1}^{N} x_{i} \omega_{i} \in \mathbb{R}^{N}$ with $x_{i} \geq 0(1 \leq i \leq N)$ then we simply write $x \geq 0$. 
Consider the surjective map $A: \mathbb{R}^{N} \rightarrow V$ defined by $A\left(\omega_{i}\right)=\alpha_{i}$ and denote by $K$ its kernel. Then $K$ is a vector space of dimension $d=N-r$ equipped with the quotient Lebesgue measure $d x / d h$.

If $h \in V$, we define

$$
\Pi_{\mathcal{A}^{+}}(h)=\left\{x \in \mathbb{R}^{N} \mid A x=h ; x \geq 0\right\} .
$$

The set $\Pi_{\mathcal{A}^{+}}(h)$ is a convex polytope. It is the intersection of the non-negative quadrant in $\mathbb{R}^{N}$ with an affine translate of the vector space $K$. This polytope consists of all nonnegative solutions of the system of $r$ linear equations

$$
\sum_{i=1}^{N} x_{i} \alpha_{i}=h .
$$

Remark 2.3. It might be appropriate to recall that any full-dimensional convex polytope $P$ in a vector space $E$ of dimension $d$, defined by a system of $N$ linear inequations

$$
P=\left\{y \in E \mid\left\langle u_{i}, y\right\rangle+\lambda_{i} \geq 0\right\}
$$

(where $u_{i} \in E^{*}$ and $\lambda_{i}$ are real numbers), can be canonically realized as a partition polytope $\Pi_{\mathcal{A}^{+}}(h)$.

If $h$ is in the interior of the cone $\mathcal{C}(\mathcal{A})$, then the polytope $\Pi_{\mathcal{A}^{+}}(h)$ is of dimension $d$. It lies in a translate of the vector space $K$, and this translated space is provided with the quotient measure $d x / d h$.

Definition 2.4. We write $\operatorname{vol}_{\mathcal{A}^{+}}(h)$ for the volume of $\Pi_{\mathcal{A}^{+}}(h)$ computed with respect to this measure.

Suppose further that $V$ is provided with a lattice $V_{\mathbb{Z}}$ and that

$$
\mathcal{A}^{+}:=\left[\alpha_{1}, \alpha_{2}, \ldots, \alpha_{N}\right]
$$

is a sequence of non-zero elements of $V_{\mathbb{Z}}$ spanning $V_{\mathbb{Z}}$, that is, $V_{\mathbb{Z}}=\sum_{i=1}^{N} \mathbb{Z} \alpha_{i}$.

In this case the lattice $V_{\mathbb{Z}}$ determines a measure $d_{\mathbb{Z}} h$ on $V$ so that the fundamental domain of the lattice $V_{\mathbb{Z}}$ is of measure 1 for $d_{\mathbb{Z}} h$. However, for reasons which will be clear later, we keep our initial measure $d h$. We introduce the normalized volume.

Definition 2.5. The normalized volume $\operatorname{vol}_{\mathbb{Z}, \mathcal{A}^{+}}(h)$ is the volume of $\Pi_{\mathcal{A}^{+}}(h)$ computed with respect to the measure $d x / d_{\mathbb{Z}} h$.

Remark 2.6. The reason for keeping our initial $d h$ is that the root systems $B_{r}, C_{r}$, and $D_{r}$ live on the same standard vector space $V=\mathbb{R}^{r}$, where the most natural measure is the standard one. This measure is twice the measure given by the root lattice in the case of $C_{r}$ and $D_{r}$. 
If $\operatorname{vol}\left(V / V_{\mathbb{Z}}, d h\right)$ is the volume of a fundamental domain of $V_{\mathbb{Z}}$ for $d h$, clearly $\operatorname{vol}_{\mathbb{Z}, \mathcal{A}^{+}}(h)=\operatorname{vol}\left(V / V_{\mathbb{Z}}, d h\right) \operatorname{vol}_{\mathcal{A}^{+}}(h)$.

Now let $h \in V_{\mathbb{Z}}$. A discrete analogue of the normalized volume of $\Pi_{\mathcal{A}^{+}}(h)$ is the number of integral points inside this polytope.

Definition 2.7. Let $N_{\mathcal{A}^{+}}(h)$ be the number of integral points in $\Pi_{\mathcal{A}^{+}}(h)$, that is the number of solutions $x=\left(x_{1}, \ldots, x_{N}\right)$ of the equation $\sum_{i=1}^{N} x_{i} \alpha_{i}=h$ where $x_{i}$ are non-negative integers. The function $h \mapsto N_{\mathcal{A}^{+}}(h)$ is called the partition function of $\mathcal{A}^{+}$.

We will see after stating Theorem 3.3 that the functions $h \mapsto \operatorname{vol}_{\mathcal{A}^{+}}(h)$ and $h \mapsto$ $N_{\mathcal{A}^{+}}(h)$ are respectively polynomial and quasipolynomial on each chamber of $\mathcal{C}(\mathcal{A})$.

The following formulas (see, for example, [24]) compute the Laplace transform of the locally polynomial function $\operatorname{vol}_{\mathcal{A}^{+}}(h)$ and the discrete Laplace transform of the quasipolynomial function $N_{\mathcal{A}^{+}}(h)$.

Proposition 2.8. $\quad$ Let $u \in \mathcal{C}(\mathcal{A})^{*}$. Then:

1. $\int_{\mathcal{C}(\mathcal{A})} e^{-\langle h, u\rangle} \operatorname{vol}_{\mathcal{A}^{+}}(h) d h=1 / \prod_{i=1}^{N} \alpha_{i}(u)$.

2. $\sum_{h \in V_{\mathbb{Z}} \cap \mathcal{C}(\mathcal{A})} e^{-\langle h, u\rangle} N_{\mathcal{A}^{+}}(h)=1 / \prod_{i=1}^{N}\left(1-e^{-\left\langle\alpha_{i}, u\right\rangle}\right)$.

\section{Jeffrey-Kirwan Residue}

The aim of this section is to explain some theoretical results due to Jeffrey and Kirwan which are fundamental for our work. They described an efficient scheme for computing the inverse Laplace transforms in the context of hyperplane arrangements.

Let us go back to the space of rational functions $\mathcal{R}_{\mathcal{A}}$. It is $\mathbb{Z}$-graded by degree. Of great importance for our exposition are certain functions in $\mathcal{R}_{\mathcal{A}}$ of degree $-r$. Every function in $\mathcal{R}_{\mathcal{A}}$ of degree $-r$ may be decomposed into a sum of basic fractions $f_{\sigma}$ (see (1)) and degenerate fractions; degenerate fractions are those for which the linear forms in the denominator do not span $V$. Given $\sigma \in \operatorname{Bases}(\mathcal{A})$, we write $\mathcal{C}(\sigma)$ for the cone generated by $\alpha_{i}(i \in \sigma)$ and by $\operatorname{vol}(\sigma)>0$ for the volume of the parallelotope $\sum_{i=1}^{r}[0,1] \alpha_{i}$ computed for the measure $d h$. Observe that $\operatorname{vol}(\sigma)=|\operatorname{det}(\sigma)|$, where $\sigma$ is the matrix whose columns are the $\alpha_{i}$ 's. Now having fixed a chamber $\mathfrak{c}$, we define a functional $\mathrm{JK}_{\mathfrak{c}}(\varphi)$ on $\mathcal{R}_{\mathcal{A}}$ called the Jeffrey-Kirwan residue (or $J K$ residue) as follows. Let

$$
\mathrm{JK}_{\mathfrak{c}}\left(f_{\sigma}\right)= \begin{cases}\operatorname{vol}(\sigma)^{-1}, & \text { if } \quad \mathfrak{c} \subset \mathcal{C}(\sigma), \\ 0, & \text { if } \quad \mathfrak{c} \cap \mathcal{C}(\sigma)=\emptyset .\end{cases}
$$

By setting the value of the $\mathrm{JK}$ residue of a degenerate fraction or that of a rational function of pure degree different from $-r$ equal to zero, we have defined the JK residue on $\mathcal{R}_{\mathcal{A}}$.

We may go further and extend the definition to the space $\widehat{\mathcal{R}}_{\mathcal{A}}$ which is the space consisting of functions $P / Q$ where $Q$ is a product of powers of the linear forms $\alpha_{i}$ and $P=\sum_{k=0}^{\infty} P_{k}$ is a formal power series. Indeed, suppose that $P / Q \in \widehat{\mathcal{R}}_{\mathcal{A}}$ where we may assume that $Q$ is of degree $q$, and $P=\sum_{k=0}^{\infty} P_{k}$ is a formal power series with $P_{k}$ of 
degree $k$. Then we just define

$$
\mathrm{JK}_{\mathfrak{c}}(P / Q)=\mathrm{JK}_{\mathfrak{c}}\left(P_{q-r} / Q\right)
$$

as the JK residue of the component of degree $-r$ of $P / Q$. In particular, if $\varphi \in \mathcal{R}_{\mathcal{A}}$ and $h \in V$, the function

$$
e^{\langle h, u\rangle} \varphi(u)=\sum_{k=0}^{\infty} \frac{\langle h, u\rangle^{k}}{k !} \varphi(u)
$$

is in $\widehat{\mathcal{R}}_{\mathcal{A}}$ and we may compute its JK residue. Observe that the JK residue depends on the measure $d h$.

Theorem 3.1 (Jeffrey-Kirwan). If $\varphi \in \mathcal{R}_{\mathcal{A}}$, then for any $h \in \mathfrak{c}$ we have

$$
\left(L^{-1} \varphi\right)(h)=\mathrm{JK}_{\mathfrak{c}}\left(e^{\langle h, \cdot\rangle} \varphi\right) .
$$

Assume that $\Psi: U \rightarrow U$ is a holomorphic transformation defined on a neighborhood of 0 in $U$ and invertible. We also assume that $\alpha_{j}(F(u))=\alpha_{j}(u) f_{j}(u)$, where $f_{j}(u)$ is holomorphic in a neighborhood of 0 and $f_{j}(0) \neq 0$.

If $\varphi$ is a function in $\widehat{\mathcal{R}}_{\mathcal{A}}$, the function $\Psi^{*} \varphi(u)=\varphi(\Psi(u))$ is again in $\widehat{\mathcal{R}}_{\mathcal{A}}$. Let $\operatorname{Jac}(\Psi)$ be the Jacobian of the map $\Psi$. For any $\varphi$ in $\widehat{\mathcal{R}}_{\mathcal{A}}$ the following change of variable formula (Theorem 45 in [1]), which will be useful in our calculations later, holds:

Proposition 3.2. The JK residue obeys the rule of change of variables:

$$
\operatorname{JK}_{\mathfrak{c}}(\varphi)=\operatorname{JK}_{\mathfrak{c}}\left(\operatorname{Jac}(\Psi)\left(\Psi^{*} \varphi\right)\right) .
$$

We conclude this section by recalling the formula for $N_{\mathcal{A}^{+}}(h)$.

Consider the dual lattice $U_{\mathbb{Z}}=\left\{u \in U \mid\left\langle u, V_{\mathbb{Z}}\right\rangle \subset \mathbb{Z}\right\}$ and the torus $T=U / U_{\mathbb{Z}}$. Choosing a basis $\left\{u_{1}, \ldots, u_{r}\right\}$ of $U_{\mathbb{Z}}$ we may identify $T$ with the subset of $U$ defined by the fundamental domain $\left\{\sum_{j=1}^{r} t_{j} u_{j}\right\}$ with $0 \leq t_{j}<1$. In this setting we use additive notation for $T$ and denote the identity by $g=0$.

Every element $g$ in $T=U / U_{\mathbb{Z}}$ produces a function on $V_{\mathbb{Z}}$ by $h \mapsto e^{\langle h, 2 \pi \sqrt{-1} G\rangle}$, where we denote by $G$ a representative of $g \in U / U_{\mathbb{Z}} \cdot{ }^{1}$ For $\sigma \in \operatorname{Bases}(\mathcal{A})$ we denote by $T(\sigma)$ the subset of $T$ defined by

$$
T(\sigma)=\left\{g \in T \mid e^{\langle\alpha, 2 \pi \sqrt{-1} G\rangle}=1 \text { for all } \alpha \in \sigma\right\} .
$$

This is a finite subset of $T$. In particular, if $\sigma$ is a $\mathbb{Z}$-basis of $V_{\mathbb{Z}}$, then $T(\sigma)$ is reduced to the identity. More generally, consider the lattice $\mathbb{Z} \sigma$ generated by the elements $\alpha$ in $\sigma$. If $p$ is an integer such that $\mathbb{Z} \sigma \subset p V_{\mathbb{Z}}$, then all elements of $T(\sigma)$ are of order $p$.

For $g \in T$ and $h \in V_{\mathbb{Z}}$, consider the Kostant function $F(g, h)$ on $U$ defined by

$$
F(g, h)(u)=\frac{e^{\langle h, 2 \pi \sqrt{-1} G+u\rangle}}{\prod_{i=1}^{N}\left(1-e^{-\left\langle\alpha_{i}, 2 \pi \sqrt{-1} G+u\right\rangle}\right)} .
$$

\footnotetext{
${ }^{1}$ We prefer to denote the complex number $i$ by $\sqrt{-1}$ because we use $i$ for many indices.
} 
For example, when $g=0$,

$$
F(0, h)(u)=\frac{e^{\langle h, u\rangle}}{\prod_{i=1}^{N}\left(1-e^{\left.-\left\langle\alpha_{i}, u\right\rangle\right)}\right.} .
$$

The function $F(g, h)(u)$ is an element of $\widehat{\mathcal{R}}_{\mathcal{A}}$. Indeed, if we write

$$
I(g)=\left\{i \mid 1 \leq i \leq N, e^{-\left\langle\alpha_{i}, 2 \pi \sqrt{-1} G\right\rangle}=1\right\},
$$

then

$$
F(g, h)(u)=e^{\langle h, 2 \pi \sqrt{-1} G\rangle} \frac{e^{\langle h, u\rangle}}{\prod_{i \in I(g)}\left\langle\alpha_{i}, u\right\rangle} \psi^{g}(u),
$$

where $\psi^{g}(u)$ is the holomorphic function of $u$ (in a neighborhood of zero) defined by

$$
\psi^{g}(u)=\prod_{i \in I(g)} \frac{\left\langle\alpha_{i}, u\right\rangle}{\left(1-e^{-\left\langle\alpha_{i}, u\right\rangle}\right)} \times \prod_{i \notin I(g)} \frac{1}{\left(1-e^{-\left\langle\alpha_{i}, 2 \pi \sqrt{-1} G+u\right\rangle}\right)} .
$$

If $\mathfrak{c}$ is a chamber of $\mathcal{C}(\mathcal{A})$, the $\mathrm{JK}$ residue $\mathrm{JK}_{\mathfrak{c}}(F(g, h))$ is well defined.

The following theorem is due to Szenes and Vergne [22]. If the set $\mathcal{A}$ is unimodular (that is, each $\sigma \in \operatorname{Bases}(\mathcal{A})$ is a $\mathbb{Z}$-basis of $V_{\mathbb{Z}}$ ), it is a reformulation of the KhovanskiiPukhlikhov Riemann-Roch calculus on simple polytopes [18]. For a general set $\mathcal{A}$, this refines the formula of Brion and Vergne [7].

Theorem 3.3. Let $\mathfrak{c}$ be a chamber of the cone $\mathcal{C}(\mathcal{A})$ and let $\overline{\mathfrak{c}}$ be its closure. Then:

1. For $h \in \overline{\mathfrak{c}}$ we have

$$
\operatorname{vol}_{\mathbb{Z}, \mathcal{A}^{+}}(h)=\operatorname{vol}\left(V / V_{\mathbb{Z}}, d h\right) \mathrm{JK}_{\mathfrak{c}}\left(\frac{e^{\langle h, \cdot\rangle}}{\prod_{i=1}^{N} \alpha_{i}}\right) .
$$

2. Assume that $\Gamma$ is a finite subset of $T$ such that for any $\sigma \in \operatorname{Bases}(\mathcal{A})$, we have $T(\sigma) \subset \Gamma$. Then for $h \in V_{\mathbb{Z}} \cap \overline{\mathfrak{c}}$, we have

$$
N_{\mathcal{A}^{+}}(h)=\operatorname{vol}\left(V / V_{\mathbb{Z}}, d h\right) \sum_{g \in \Gamma} \mathrm{JK}_{\mathfrak{c}}(F(g, h)) .
$$

Observe that the right-hand side of the equation in condition 2 does not depend on the measure $d h$, as it should.

Remark 3.4. If $\mathcal{A}$ is unimodular, we can take $\Gamma:=\{0\}$.

Let us explain the behavior of these functions on a chamber $\mathfrak{c}$. By definition, a quasipolynomial function on a lattice $L$ is a linear combination of products of polynomial functions and of periodic functions (functions constant on cosets $h+p L$ where $p$ is an integer). We now show that the normalized volume $\operatorname{vol}_{\mathbb{Z}, \mathcal{A}^{+}}(h)$ is given by a polynomial formula, when $h$ varies in a chamber $\overline{\mathfrak{c}}$, while $N_{\mathcal{A}^{+}}(h)$ is given by a quasipolynomial formula when $h$ varies in $V_{\mathbb{Z}} \cap \overline{\mathfrak{c}}$. 
The residue vanishes except on degree $-r$, so that

$$
\mathrm{JK}_{\mathfrak{c}}\left(\frac{e^{\langle h, u\rangle}}{\prod_{i=1}^{N}\left\langle\alpha_{i}, u\right\rangle}\right)=\frac{1}{(N-r) !} \mathrm{JK}_{\mathfrak{c}}\left(\frac{\langle h, u\rangle^{N-r}}{\prod_{i=1}^{N}\left\langle\alpha_{i}, u\right\rangle}\right),
$$

and as expected the normalized volume is a polynomial homogeneous function of $h$ of degree $N-r$ on each chamber.

Let us analyze the behavior of the function $N_{\mathcal{A}^{+}}(h)$ expressing the number of integral points.

Let $g \in T$ and denote by $\psi^{g}(u)=\sum_{k=0}^{+\infty} \psi_{k}^{g}(u)$ the series development of the holomorphic function $\psi^{g}$ appearing in formula (4). Then we see that $\operatorname{JK}_{\mathfrak{c}}(F(g, h))$ equals

$$
\begin{aligned}
\mathrm{JK}_{\mathfrak{c}}\left(e^{\langle h, 2 \pi \sqrt{-1} G\rangle} \frac{e^{\langle h, u\rangle}}{\prod_{i \in I(g)}\left\langle\alpha_{i}, u\right\rangle} \psi^{g}(u)\right) \\
=e^{\langle h, 2 \pi \sqrt{-1} G\rangle} \sum_{k=0}^{|I(g)|-r} \frac{1}{(|I(g)|-r-k) !} \mathrm{JK}_{\mathfrak{c}}\left(\frac{\langle h, u\rangle^{|I(g)|-r-k}}{\prod_{i \in I(g)}\left\langle\alpha_{i}, u\right\rangle} \psi_{k}^{g}(u)\right) .
\end{aligned}
$$

If $g$ is of order $p$, the function $h \mapsto e^{\langle h, 2 \pi \sqrt{-1} G\rangle}$ is constant on each coset $h+p V_{\mathbb{Z}}$ of the lattice $p V_{\mathbb{Z}}$, while the function $h \mapsto \mathrm{JK}_{\mathfrak{c}}\left(\left(\langle h, u\rangle^{|I(g)|-r-k} / \prod_{i \in I(g)}\left\langle\alpha_{i}, u\right\rangle\right) \psi_{k}^{g}(u)\right)$ is a polynomial function of $h$ of degree $|I(g)|-r-k$. Thus the function

$$
N_{\mathcal{A}^{+}}(h)=\operatorname{vol}\left(V / V_{\mathbb{Z}}, d h\right) \sum_{g \in \Gamma} \operatorname{JK}_{\mathfrak{c}}(F(g, h))
$$

is given by a quasipolynomial formula when $h$ varies in the closure of a chamber. In particular, if $\mathcal{A}$ is unimodular (like in the case of the positive root system of $A_{r}$ ), then $\Gamma$ is reduced to the identity and $N_{\mathcal{A}^{+}}(h)$ is polynomial. In the non-unimodular case (like in the case of the positive root system of $B_{r}, C_{r}, D_{r}$ ), the set $\Gamma$ is not reduced to the identity, and the function $N_{\mathcal{A}^{+}}(h)$ is in general only quasipolynomial.

Note that its highest degree component of $N_{\mathcal{A}^{+}}(h)$ is polynomial and is the normalized volume as expected.

Example 3.5. Let us consider the root system $B_{2}$, that is for $\mathcal{A}^{+}=\mathcal{B}_{2}=\left\{e_{1}, e_{2}, e_{1}+\right.$ $\left.e_{2}, e_{1}-e_{2}\right\}$. Fix a chamber $c$ and an integral vector $h=\left(h_{1}, h_{2}\right)$ in the cone $\mathcal{C}\left(\mathcal{B}_{2}\right)$. Observe that the root lattice is $\mathbb{Z} e_{1} \oplus \mathbb{Z} e_{2}$ and $\operatorname{vol}\left(V / V_{\mathbb{Z}}, d h\right)=1$ for the measure $d h=d h_{1} d h_{2}$. There are three chambers, namely $\mathfrak{c}_{1}=\mathcal{C}\left(\left\{e_{2}, e_{1}+e_{2}\right\}\right), \mathfrak{c}_{2}=\mathcal{C}\left(\left\{e_{1}, e_{1}+e_{2}\right\}\right)$, and $\mathfrak{c}_{3}=\mathcal{C}\left(\left\{e_{1}-e_{2}, e_{1}\right\}\right)$ (see Fig. 3). Following the procedure explained above (see formulas (6) and (7)) and computing the JK residues on the chambers we obtain:

\begin{tabular}{ccc}
\hline $\operatorname{vol}\left(\Pi_{\mathcal{B}_{2}}(h)\right)$ & $N_{\mathcal{B}_{2}}(h)$ & Chamber \\
\hline$\frac{1}{2} h_{1}^{2}$ & $1+\frac{3}{2} h_{1}+\frac{1}{2} h_{1}^{2}$ & $\mathfrak{c}_{1}$ \\
$\frac{1}{4}\left(h_{1}+h_{2}\right)^{2}-\frac{1}{2} h_{2}^{2}$ & $\frac{1}{4} h_{1}^{2}+\frac{1}{2} h_{1} h_{2}-\frac{1}{4} h_{2}^{2}+h_{1}+\frac{1}{2} h_{2}+\frac{7}{8}+(-1)^{h_{1}+h_{2}} \frac{1}{8}$ & $\mathfrak{c}_{2}$ \\
$\frac{1}{4}\left(h_{1}+h_{2}\right)^{2}$ & $\frac{1}{4} h_{1}^{2}+\frac{1}{2} h_{1} h_{2}+\frac{1}{4} h_{2}^{2}+h_{1}+h_{2}+\frac{7}{8}+(-1)^{h_{1}+h_{2}} \frac{1}{8}$ & $\mathfrak{c}_{3}$ \\
\hline
\end{tabular}




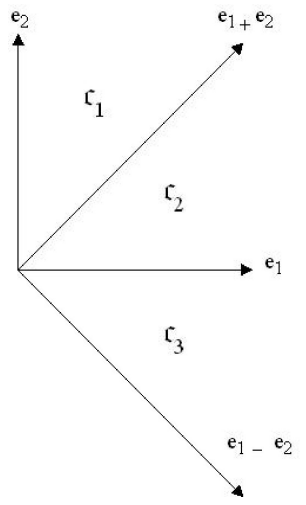

Fig. 3. The three chambers for $B_{2}$.

Note that the formulas agree on walls $\mathfrak{c}_{1} \cap \mathfrak{c}_{2}$ and $\mathfrak{c}_{2} \cap \mathfrak{c}_{3}$ and they are valid on the closures of the chambers.

Remark 3.6. Combining (6) and (7), we can see that the quasipolynomial character of the integral-point counting functions $N_{A}^{+}$stems precisely from the root of unity in (6). Furthermore, we will see in Lemmas 8.2, 9.1, and 10.1 that for root systems of type $B$, $C$, and $D$, these roots of unity are of order 2 , as in the above example for $\mathcal{B}_{2}$. Let us record the following immediate consequence:

\section{Corollary 3.7.}

- The integral-point counting functions $N_{\mathcal{A}_{r}}$ is polynomial.

- The integral-point counting functions $N_{\mathcal{B}_{r}}, N_{\mathcal{C}_{r}}, N_{\mathcal{D}_{r}}$ are quasipolynomials with period 2.

Remark 3.8. The partition functions $N_{\mathcal{A}_{r}}, N_{\mathcal{B}_{r}}, N_{\mathcal{C}_{r}}, N_{\mathcal{D}_{r}}$ can be interpreted as (weak) flow quasipolynomials on certain signed graphs [5]. The polynomiality of $N_{\mathcal{A}_{r}}$ follows immediately from this interpretation and a unimodularity argument; the fact that the quasipolynomials $N_{\mathcal{B}_{r}}, N_{\mathcal{C}_{r}}, N_{\mathcal{D}_{r}}$ have period 2 follows from a half-integrality result of Lee [19].

Remark 3.9. In the case where $\mathcal{A}^{+}$is an arbitrary sequence of vectors in $V_{\mathbb{Z}}$, the straightforward implementation of Theorem 3.3 above is of exponential complexity. Indeed, we make a summation on the set $\Gamma$, which can become arbitrarily large. Barvinok uses a signed cone decomposition to obtain an algorithm of polynomial complexity, when the number of elements of $\mathcal{A}^{+}$is fixed, to compute the number $N_{\mathcal{A}^{+}}(h)$; the LattE team implemented Barvinok's algorithm [14], [15] in the language C. Our work will be dealing either with volumes of polytopes, where the set $\Gamma$ does not enter, or with the partition function of classical root systems, where the set $\Gamma$ is reasonably small. Then we obtain a fast algorithm, implemented for the moment in the formal calculation software MAPLE. 
This algorithm for these particular cases can reach examples not obtainable by the Latt $\mathrm{E}$ program.

\section{A Formula for the Jeffrey-Kirwan Residue}

If $f$ is a meromorphic function of one variable $z$ with a pole of order less than or equal to $h$ at $z=0$ then we can write $f(z)=Q(z) / z^{h}$, where $Q(z)$ is a holomorphic function near $z=0$. If the Taylor series of $Q$ is given by $Q(z)=\sum_{k=0}^{\infty} q_{k} z^{k}$, then as usual the residue at $z=0$ of the function $f(z)=\sum_{k=0}^{\infty} q_{k} z^{k-h}$ is the coefficient of $1 / z$, that is, $q_{h-1}$. We denote it by res $\operatorname{res}_{z=0} f(z)$. To compute this residue we can either expand $Q$ into a power series and search for the coefficient of $z^{-1}$, or employ the formula

$$
\operatorname{res}_{z=0} f(z)=\left.\frac{1}{(h-1) !}\left(\partial_{z}\right)^{h-1}\left(z^{h} f(z)\right)\right|_{z=0} .
$$

We now introduce the notion of iterated residue on the space $\mathcal{R}_{\mathcal{A}}$.

Let $\vec{v}=\left[\alpha_{1}, \alpha_{2}, \ldots, \alpha_{r}\right]$ be an ordered basis of $V$ consisting of elements of $\mathcal{A}$ (here we have implicitly renumbered the elements of $\mathcal{A}$ in order that the elements of our basis are listed first). We choose a system of coordinates on $U$ such that $\alpha_{i}(u)=$ $u_{i}$. A function $\varphi \in \mathcal{R}_{\mathcal{A}}$ is thus written as a rational fraction $\varphi\left(u_{1}, u_{2}, \ldots, u_{r}\right)=$ $P\left(u_{1}, u_{2}, \ldots, u_{r}\right) / Q\left(u_{1}, u_{2}, \ldots, u_{r}\right)$ where the denominator $Q$ is a product of linear forms.

Definition 4.1. If $\varphi \in \mathcal{R}_{\mathcal{A}}$, the iterated residue $\operatorname{Ires}_{\vec{v}}(\varphi)$ of $\varphi$ for $\vec{v}$ is the scalar

$$
\operatorname{Ires}_{\vec{v}}(\varphi)=\operatorname{res}_{u_{r}=0} \operatorname{res}_{u_{r-1}=0} \cdots \operatorname{res}_{u_{1}=0} \varphi\left(u_{1}, u_{2}, \ldots, u_{r}\right),
$$

where each residue is taken assuming that the variables with higher indices are considered constants.

Observe that the value of $\operatorname{Ires}_{\vec{v}}(\varphi)$ depends on the order of $\vec{v}$. For example, for $f=$ $1 /(x(y-x))$ we have $\operatorname{res}_{x=0} \operatorname{res}_{y=0}(f)=0$ and $\operatorname{res}_{y=0} \operatorname{res}_{x=0}(f)=1$.

Remark 4.2. Choose any basis $\gamma_{1}, \gamma_{2}, \ldots, \gamma_{r}$ of $V$ such that $\bigoplus_{k=1}^{j} \alpha_{j}=\bigoplus_{k=1}^{j} \gamma_{j}$ for every $1 \leq j \leq r$ and such that $\gamma_{1} \wedge \gamma_{2} \wedge \cdots \wedge \gamma_{r}=\alpha_{1} \wedge \alpha_{2} \wedge \cdots \wedge \alpha_{r}$. Then, by induction, it is easy to see that for $\varphi \in \mathcal{R}_{\mathcal{A}}$,

$$
\operatorname{res}_{\alpha_{r}=0} \cdots \operatorname{res}_{\alpha_{1}=0} \varphi=\operatorname{res}_{\gamma_{r}=0} \cdots \operatorname{res}_{\gamma_{1}=0} \varphi .
$$

Thus given an ordered basis, we may modify $\alpha_{2}$ by $\alpha_{2}+c \alpha_{1}, \ldots$, with the purpose of getting easier computations.

The following lemma will be useful later.

Lemma 4.3. Let $\vec{v}=\left[\alpha_{1}, \alpha_{2}, \ldots, \alpha_{r}\right]$ and let $f_{\beta}=1 / \prod_{i=1}^{r} \beta_{i}$ be a basic fraction. Then the iterated residue $\operatorname{Ires}_{\vec{v}}\left(f_{\beta}\right)$ is non-zero if and only if there exists a permutation $w$ of $\{1,2, \ldots, r\}$ such that

$$
\beta_{w(i)} \in \mathbb{R} \alpha_{1} \oplus \cdots \oplus \mathbb{R} \alpha_{i} \quad \text { for all } \quad 1 \leq i \leq r .
$$


Definition 4.4. Let $\vec{v}=\left[\alpha_{1}, \alpha_{2}, \ldots, \alpha_{r}\right]$ and let $u_{j}=\alpha_{j}(u)$. Choose a sequence of real numbers: $0<\varepsilon_{1}<\varepsilon_{2}<\cdots<\varepsilon_{r}$. Then define the torus

$$
T(\vec{v})=\left\{u \in U_{\mathbb{C}}|| u_{j} \mid=\varepsilon_{j}, j=1, \ldots, r\right\} .
$$

The torus $T(\vec{v})$ is identified via the basis $\alpha_{j}$ with the product of $r$ circles oriented counterclockwise. The sequence $\left[\varepsilon_{1}, \varepsilon_{2}, \ldots, \varepsilon_{r}\right]$ is chosen so that elements $\alpha_{q}$ not in $\bigoplus_{k=1}^{j} \mathbb{R} \alpha_{j}$ do not vanish on the domain $\left\{u \in U_{\mathbb{C}}|| u_{k}\left|\leq \varepsilon_{k}, 1 \leq k \leq j ;\right| u_{i} \mid=\varepsilon_{i}, i=\right.$ $j+1, \ldots, r\}$. This is achieved by choosing the ratios $\varepsilon_{j} / \varepsilon_{j+1}$ very small. The torus $T(\vec{v})$ is contained in $U_{\mathbb{C}}(\mathcal{A})$ and the homology class $[T(\vec{v})]$ of this torus is independent of the choice of the sequence of the ordered $\varepsilon_{j}$ [23].

Choose an ordered basis $e_{1}, e_{2}, \ldots, e_{r}$ of $V$ of volume 1 with respect to the measure $d h$. For $z \in U_{\mathbb{C}}$, define $z_{j}=\left\langle z, e_{j}\right\rangle$ and $d z=d z_{1} \wedge d z_{2} \wedge \cdots \wedge d z_{r}$. Denote by $\operatorname{det}(\vec{v})$ the determinant of the basis $\alpha_{1}, \alpha_{2}, \ldots, \alpha_{r}$ with respect to the basis $e_{1}, e_{2}, \ldots, e_{r}$.

Lemma 4.5. For $\varphi \in \mathcal{R}_{\mathcal{A}}$, we have

$$
\frac{1}{\operatorname{det}(\vec{v})} \operatorname{res}_{\alpha_{r}=0} \cdots \operatorname{res}_{\alpha_{1}=0} \varphi=\frac{1}{(2 \pi \sqrt{-1})^{r}} \int_{T(\vec{v})} \varphi(z) d z .
$$

Thus, as for the usual residue, the iterated residue can be expressed as an integral.

We now introduce the notion, due to De Concini and Procesi [13], of a maximal proper nested set, MPNS in short.

If $S$ is a subset of $\mathcal{A}$, we denote by $\langle S\rangle$ the vector space spanned by $S$. More generally, if $M=\left\{S_{i}\right\}$ is a set of subsets of $\mathcal{A}$, we denote by $\langle M\rangle$ the vector space spanned by all elements of the sets $S_{i}$. We say that a subset $S$ of $\mathcal{A}$ is complete if $S=\langle S\rangle \cap \mathcal{A}$ or in other words if any element of $\mathcal{A}$ which is a linear combination of elements of $S$ belongs to $S$. A complete subset $S$ is called reducible if we can find a decomposition $V=V_{1} \oplus V_{2}$ such that $S=S_{1} \cup S_{2}$ with $S_{1} \subset V_{1}$ and $S_{2} \subset V_{2}$. Otherwise $S$ is said to be irreducible.

Definition 4.6. Let $\mathcal{I}$ be the set of irreducible subsets of $\mathcal{A}$. A set $M=\left\{I_{1}, I_{2}, \ldots, I_{k}\right\}$ of irreducible subsets of $\mathcal{A}$ is called nested if, given any subfamily $\left\{I_{1}, \ldots, I_{m}\right\}$ of $M$ such that there exists no $i, j$ with $I_{i} \subset I_{j}$, then the set $I_{1} \cup \cdots \cup I_{m}$ is complete and the elements $I_{j}$ are the irreducible components of $I_{1} \cup I_{2} \cup \cdots \cup I_{m}$.

Example 4.7. Let $E$ be an $(r+1)$-dimensional vector space with basis $e_{i}(i=1, \ldots$, $r$ ). We consider the set

$$
\mathcal{K}_{r+1}=\left\{e_{i}-e_{j} \mid 1 \leq i<j \leq r+1\right\} .
$$

These are the positive roots for the system $A_{r}$. The irreducible subsets of $\mathcal{K}_{r+1}$ are indexed by subsets $S$ of $\{1,2, \ldots, r+1\}$, the corresponding irreducible subset being $\left\{e_{i}-e_{j} \mid i, j \in S, i<j\right\}$. For instance, the set $S=\{1,2,4\}$ parametrizes the set of roots given by $\left\{e_{1}-e_{2}, e_{2}-e_{4}, e_{1}-e_{4}\right\}$.

A nested set is represented by a collection $M=\left\{S_{1}, S_{2}, \ldots, S_{k}\right\}$ of subsets of $\{1,2, \ldots, r+1\}$ such that if $S_{i}, S_{j} \in M$ then either $S_{i} \cap S_{j}$ is empty, or one of them is contained in another. 
Definition 4.8. A maximal nested set (in short MNS) $M$ is a nested set such that for every irreducible set $\mathcal{I}$ of $\mathcal{A}$ the set $M \cup\{\mathcal{I}\}$ is no longer nested.

An MNS has exactly $r$ elements [13].

Assume now that $\mathcal{A}$ is irreducible, otherwise just take the irreducible components. Then every MNS $M$ contains $\mathcal{A}$. Let $I_{1}, I_{2}, \ldots, I_{k}$ be the maximal elements of the set $M \backslash \mathcal{A}$. We see that the vector space $\left\langle I_{1}\right\rangle \oplus\left\langle I_{2}\right\rangle \oplus \cdots \oplus\left\langle I_{k}\right\rangle$ is of codimension 1 [13, Proposition 1.3].

Definition 4.9. A hyperplane $H$ in $V$ is $\mathcal{A}$-admissible if it is spanned by a set of vectors of $\mathcal{A}$.

Thus if $M$ is an MNS, the vector space $\langle M \backslash \mathcal{A}\rangle$ is an admissible hyperplane $H$.

Definition 4.10. Let $\mathcal{A}$ be irreducible and let $H$ be an $\mathcal{A}$-admissible hyperplane. All MNSs such that $\langle M \backslash \mathcal{A}\rangle=H$ are said to be attached to $H$.

Therefore to classify MNSs for an irreducible set $\mathcal{A}$ we proceed by running over the set of $\mathcal{A}$-admissible hyperplanes, as described in Fig. 4.

We now describe the notion of an MPNS of $\mathcal{A}$.

Fix a total order on the set $\mathcal{A}$. For example, we can choose a linear functional ht on $V$ so that the values $\operatorname{ht}\left(\alpha_{i}\right)$ are all distinct and positive. Thus the value ht $(\alpha)$ is larger if $\alpha$ is deeper in the interior of the cone.

Let $M=\left\{S_{1}, S_{2}, \ldots, S_{k}\right\}$ be a set of subsets of $\mathcal{A}$. In each $S_{j}$ we choose the element $\alpha_{j}$ maximal for the order given by ht. This defines a map $\theta$ from $M$ to $\mathcal{A}$.

Definition 4.11. An MNS $M$ is called proper if $\theta(M)$ is a basis of $V$. We denote by $\mathcal{P}(\mathcal{A})$ the set of MPNSs.

If $M=\left\{I_{1}, I_{2}, \ldots, I_{r}\right\}$ is an MNS, we associate to $M$ the list $\left[\theta\left(I_{i_{1}}\right), \ldots, \theta\left(I_{i_{r}}\right)\right]$ using the total order on the elements $\theta(M)$; that is we have $\operatorname{ht}\left(\theta\left(I_{i_{1}}\right)\right)<\operatorname{ht}\left(\theta\left(I_{i_{2}}\right)\right)<$ $\cdots<\operatorname{ht}\left(\theta\left(I_{i_{r}}\right)\right)$. Observe that, if $\mathcal{A}$ is irreducible, for every MNS, $I_{i_{r}}$ is always equal to $\mathcal{A}$ and $\theta\left(I_{i_{r}}\right)$ is the highest element of $\mathcal{A}$. We often implicitly renumber our elements in $M$ such that ht $\left(\theta\left(I_{1}\right)\right)<\operatorname{ht}\left(\theta\left(I_{2}\right)\right)<\cdots<\operatorname{ht}\left(\theta\left(I_{r}\right)\right)$.

We have associated to every MPNS $M$ an ordered basis $\overrightarrow{\theta(M)}=\left[\alpha_{1}, \alpha_{2}, \ldots, \alpha_{r}\right]$ of elements of $\mathcal{A}$. We denote by $\operatorname{vol}(M)>0$ the volume of the parallelepiped $\sum_{i=1}^{r}[0,1] \alpha_{i}$ with respect to our measure, and by $\mathcal{C}(M)=\sum_{i=1}^{r} \mathbb{R}_{\geq 0} \alpha_{i} \subset \mathcal{C}(\mathcal{A})$ the cone generated by $\theta(M)$.

- Take a hyperplane $H$ spanned by a set of vectors of $\mathcal{A}$.

- Break $\mathcal{A} \cap H$ into irreducible subsets $I_{1} \cup I_{2} \cup \cdots \cup I_{k}$.

- For each irreducible $I_{i}$ construct the set $\left\{M_{1}^{i}, \ldots, M_{k_{i}}^{i}\right\}$ of MNSs for $I_{i}$.

- Set $C_{i}=\left\{1, \ldots, k_{i}\right\}$.

- An MNS is then given by the union $M_{c_{1}}^{1} \cup M_{c_{2}}^{2} \cup \cdots \cup M_{c_{k}}^{k} \cup\{\mathcal{A}\}$ where $c_{1} \in C_{1}, \ldots$, $c_{k} \in C_{k}$, and all of them are obtained by letting $c_{i}$ vary.

Fig. 4. Building of all MNSs attached to an $\mathcal{A}$-admissible hyperplane $H$. 
If $v$ is a regular element of $V$, let

$$
\mathcal{P}(v, \mathcal{A})=\{M \in \mathcal{P}(\mathcal{A}) \mid v \in \mathcal{C}(M)\} .
$$

The set $\mathcal{P}(v, \mathcal{A})$ depends only of the chamber $\mathfrak{c}$ where $v$ belongs. We are now ready to state the basic formula for our calculations.

Theorem 4.12 [13]. Let $\mathfrak{c}$ be a chamber and let $v \in \mathfrak{c}$. Then, for $\varphi \in \mathcal{R}_{\mathcal{A}}$, we have

$$
\mathrm{JK}_{\mathfrak{c}}(\varphi)=\sum_{M \in \mathcal{P}(v, \mathcal{A})} \frac{1}{\operatorname{vol}(M)} \operatorname{Ires} \overline{\theta(M)} \varphi .
$$

We also use the corresponding integration formula.

Each MPNS $M \in \mathcal{P}(v, \mathcal{A})$ determines an oriented cycle $[T(\overrightarrow{\theta(M)})]$ contained in the open set $U_{\mathbb{C}}(\mathcal{A})$, as described in Definition 4.4.

Definition 4.13. Let $\mathfrak{c}$ be a chamber. Define the oriented cycle:

$$
H(\mathfrak{c})=\sum_{M \in \mathcal{P}(v, \mathcal{A})} \operatorname{sign}(\operatorname{det}(\overrightarrow{\theta(M)}))[T(\overrightarrow{\theta(M)})]
$$

The following integral version of Theorem 4.12 will be useful.

Theorem 4.14. Let $\mathfrak{c}$ be a chamber. Then for $\varphi \in \mathcal{R}_{\mathcal{A}}$ we have

$$
\mathrm{JK}_{\mathfrak{c}}(\varphi)=\frac{1}{(2 \pi \sqrt{-1})^{r}} \int_{H(\mathfrak{c})} \varphi(z) d z
$$

The following example should help clarify the notions introduced.

Example 4.15. We consider the set $\mathcal{K}_{4}$ of positive roots for $A_{3}$ (see Fig. 5) defined by

$$
\mathcal{K}_{4}=\left\{e_{i}-e_{j} \mid 1 \leq i<j \leq 4\right\} .
$$

We let $V$ be the vector space generated by the elements in $\mathcal{K}_{4}$. Then $V$ has dimension 3 and we write an element of $V$ as

$$
a=a_{1} e_{1}+a_{2} e_{2}+a_{3} e_{3}-\left(a_{1}+a_{2}+a_{3}\right) e_{4} .
$$

We consider the height function defined by

$$
\operatorname{ht}\left(e_{1}-e_{2}\right)=10, \quad \operatorname{ht}\left(e_{2}-e_{3}\right)=11, \quad \operatorname{ht}\left(e_{3}-e_{4}\right)=12 .
$$

This choice gives the following order on the roots:

$$
e_{1}-e_{2}<e_{2}-e_{3}<e_{3}-e_{4}<e_{1}-e_{3}<e_{2}-e_{4}<e_{1}-e_{4}
$$

Take a hyperplane $H$ in $V$ spanned by two linearly independent elements of $\mathcal{K}_{4}$. Therefore it is the kernel of a linear form $\sum_{i \in I_{H}} a_{i}$, where $I_{H}$ is a proper subset of $\{1,2,3,4\}$. The set 


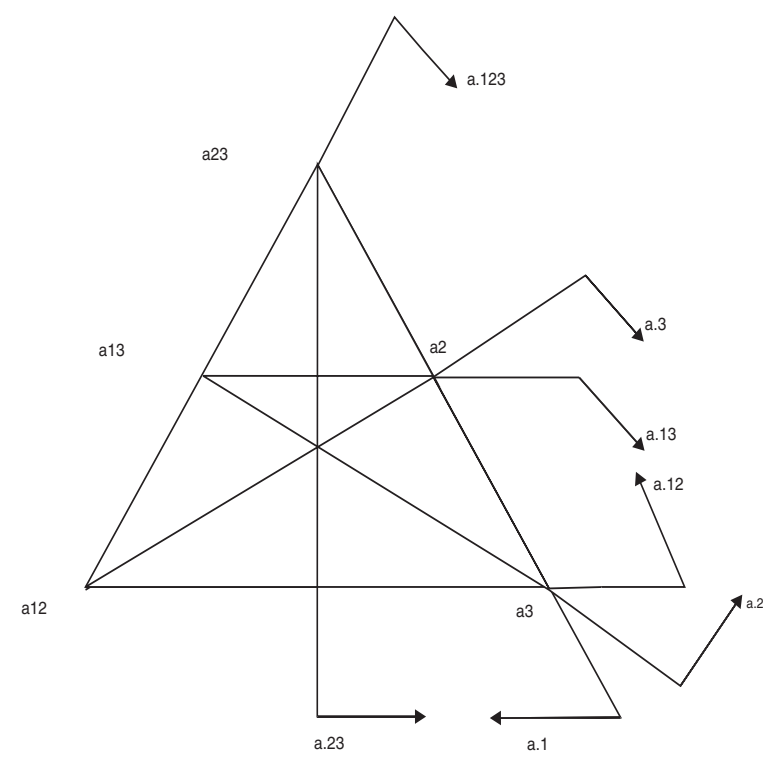

Fig. 5. Hyperplanes for $A_{3}$ with $a=a_{1} e_{1}+a_{2} e_{2}+a_{3} e_{3}-\left(a_{1}+a_{2}+a_{3}\right) e_{4}$.

of complementary indices gives the same hyperplane. Thus each admissible hyperplane partitions the set of indices $\{1,2,3,4\}$ in two sets $Z_{1}$ and $Z_{2}$, where $Z_{1}:=\left\{i \in I_{H}\right\}$ and $Z_{2}$ is the set of complementary indices. In our example we have seven choices of admissible hyperplanes corresponding to the following partitions:

$$
\begin{aligned}
& H_{1}=\{[1,2,3],[4]\}, \quad H_{2}=\{[1,2,4],[3]\}, \quad H_{3}=\{[1,3,4],[2]\}, \\
& H_{4}=\{[2,3,4],[1]\}, \quad H_{5}=\{[1,2],[3,4]\}, \quad H_{6}=\{[1,3],[2,4]\}, \\
& H_{7}=\{[1,4],[2,3]\} \text {. }
\end{aligned}
$$

Now observe that if the hyperplane $H_{i}$ already contains the highest root $e_{1}-e_{4}$ then it cannot lead to an MPNS. Indeed, we must get a basis if we add the highest root to a set of vectors contained in $H_{i}$. Thus $H_{2}, H_{3}$, and $H_{7}$ can be excluded. It remains to consider the hyperplanes $H_{1}, H_{4}, H_{5}$, and $H_{6}$.

Hyperplanes $H_{1}$ and $H_{4}$ give rise to two MPNSs each, while $H_{5}$ and $H_{6}$ give rise to only one. So we obtain a list of six MPNSs (as described in Example 4.7, we identify an irreducible subset $I$ with a subset $S$ of $[1,2,3,4])$ :

$$
\begin{array}{ll}
M_{1}=\{[1,2],[1,2,3],[1,2,3,4]\}, & M_{2}=\{[2,3],[1,2,3],[1,2,3,4]\}, \\
M_{3}=\{[2,3],[2,3,4],[1,2,3,4]\}, & M_{4}=\{[3,4],[2,3,4],[1,2,3,4]\}, \\
M_{5}=\{[1,3],[2,4],[1,2,3,4]\}, & M_{6}=\{[1,2],[3,4],[1,2,3,4]\} .
\end{array}
$$

\section{Search for Maximal Proper Nested Sets Adapted to a Vector: The General Case}

Given a vector $v$ in the cone $\mathcal{C}(\mathcal{A})$, we describe how to search for all MPNSs belonging to $\mathcal{P}(v, \mathcal{A})$, without enumerating all MPNSs. 
We use as height function a linear form that is positive and that takes different values on all elements $\alpha_{i}$, and consider the total order it induces. Let $H$ be an $\mathcal{A}$-admissible hyperplane in $V$. Then the cone $\mathcal{C}(\mathcal{A} \cap H)$ generated by the elements of $\mathcal{A}$ belonging to $H$ is a cone with non-empty interior in $H$.

We have already seen that to list all the MPNSs, we first have to list all admissible hyperplanes $H$ and then find the irreducible components $J_{1}, J_{2}, \ldots, J_{s}$ of $\mathcal{A} \cap H$. Then we choose an MPNS $M_{i}:=\left\{I_{i}^{a}\right\}$ for $J_{i}$, and define $M=M_{1} \cup M_{2} \cup M_{3} \cup \cdots \cup M_{s} \cup\{\mathcal{A}\}$.

As we have seen in Example 4.15 we can discard some of the hyperplanes a priori, because they cannot lead to an MPNS. The next lemma examines the general situation. Let $\theta$ be the highest element in $\mathcal{A}$ and let $H$ be an admissible hyperplane of $\mathcal{A}$.

Lemma 5.1. There exists an MPNS $M \in \mathcal{P}(v, \mathcal{A})$ attached to $H$, if and only if $\theta$ does not belong to $H$ and if $v$ belongs to the cone generated by $\theta$ and $\mathcal{A} \cap H$.

Proof. The condition is necessary. Indeed, $v$ must belong to the cone generated by the elements $\theta\left(I_{i}^{a}\right)$ and $\theta$, and all the elements $\theta\left(I_{i}^{a}\right)$ are in $\mathcal{A} \cap H$. Reciprocally consider the projection $v-(\langle u, v\rangle /\langle u, \theta\rangle) \theta$, where $u$ is the equation of the hyperplane $H$. This can be written as $v_{1} \oplus v_{2} \oplus \cdots \oplus v_{s}$, where each $v_{i}$ is in the cone $\mathcal{C}\left(J_{i}\right)$. Now let $M_{i} \in \mathcal{P}\left(v_{i}, J_{i}\right)$ be an MPNS in $J_{i}$. The element $v_{i}$ belongs to $\mathcal{C}\left(\theta\left(M_{i}\right)\right)$. We can write

$$
v=t \theta+\sum_{i=1}^{s} \sum_{I_{i}^{a} \in M_{i}} t_{i}^{a} \theta\left(I_{i}^{a}\right)
$$

with $t_{i}^{a}>0$. Thus we see that the collection $M_{1} \cup \cdots \cup M_{s} \cup \mathcal{A}$ is an MPNS in $\mathcal{P}(v, \mathcal{A})$. Moreover, in this way we list all elements of $\mathcal{P}(v, \mathcal{A})$.

Our search for MPNS in $\mathcal{P}(v, \mathcal{A})$ will then be pursued by constructing all possible admissible hyperplanes $H$ for which $v$ is in the convex hull of $\mathcal{C}(\mathcal{A} \cap H)$ and $\theta$. We denote by $\operatorname{Hyp}(v, \mathcal{A})$ the set of such $\mathcal{A}$-admissible hyperplanes.

The following easy lemma lists some obvious conditions for the set $\operatorname{Hyp}(v, \mathcal{A})$. Let $u_{H} \in U$ be the normal vector to an $\mathcal{A}$-admissible hyperplane, meaning that $H:=\{h \in$ $\left.V \mid\left\langle u_{H}, v\right\rangle=0\right\}$.

Lemma 5.2. If $H \in \operatorname{Hyp}(v, \mathcal{A})$ then $H$ satisfies the following conditions:

1. $\left\langle u_{H}, \theta\right\rangle \neq 0$.

2. $\left\langle u_{H}, v\right\rangle \times\left\langle u_{H}, \theta\right\rangle \geq 0$.

Thus if a hyperplane $H$ satisfies the above conditions we define

$$
\operatorname{proj}_{H}(v)=v-\frac{\left\langle u_{H}, v\right\rangle}{\left\langle u_{H}, \theta\right\rangle} \theta
$$

Hence to decide if $H \in \operatorname{Hyp}(v, \mathcal{A})$ we simply have to test if $\operatorname{proj}_{H}(v)$ is in the cone generated by $\mathcal{A} \cap H$, which is done by standard methods.

We summarize the scheme of the algorithm in Fig. 6. Recall that we have as input a vector $v$, and as output the list of all MPNSs belonging to $\mathcal{P}(v, \mathcal{A})$. 


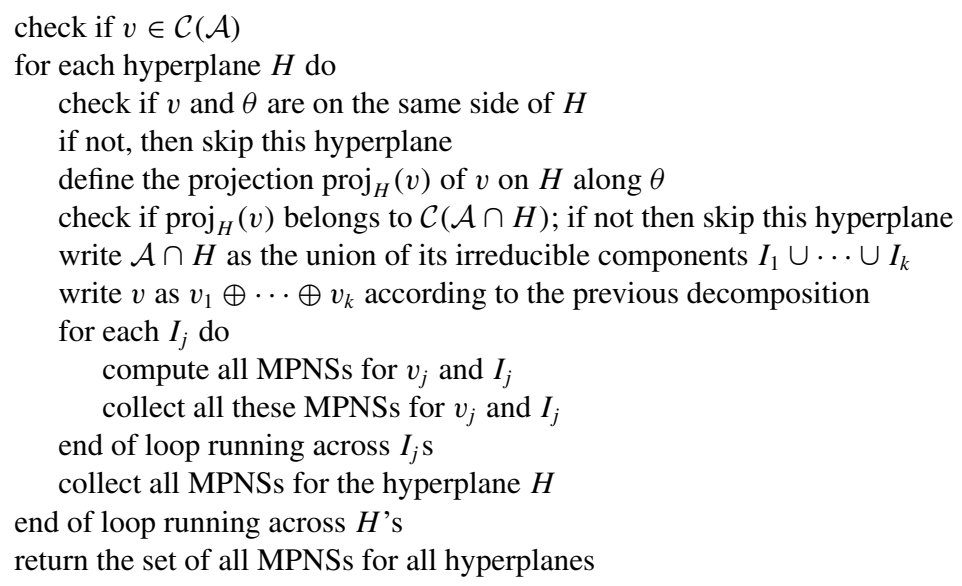

Fig. 6. Algorithm for MPNSs computation (general case).

We will explain our algorithm in more detail for each classical root system (see Sections 7-10).

\section{Trees and Order of Poles}

Let $M$ be an MNPS for the system $\mathcal{A}:=\left\{\alpha_{1}, \alpha_{2}, \ldots, \alpha_{n}\right\}$. In our algorithms we will need to take an iterated residue with respect to a basis $\overrightarrow{\theta(M)}$ of a function of the form $\varphi=$ $P / \prod_{i=1}^{N} \alpha_{i}$, where $P$ is a polynomial function on $U$. It is thus important to understand the order of the poles of the function obtained after performing a certain number of residues. We also prove that the iterated residue associated to $M$ depends only on the tree associated to $M$.

We associate to an MNS $M$ a tree $T$ as follows. Let $M=\left\{I_{1}, \ldots, I_{r}\right\}$ be an MNS. The vertices of $T$ are the elements of $M$ and the oriented edges are determined from the reverse order relation by inclusion: the ends of the tree are irreducible sets with just one element and if $\mathcal{A}$ is irreducible, the base is the set $\mathcal{A}$. A subset $N$ of $M$ is called saturated if it contains all elements above elements of $N$ in the tree order. Thus if $N$ contains an element $S$, it contains all the elements $S^{\prime}$ of $M$ which are contained in $S$.

Example 6.1. The two MNSs named $M_{1}$ and $M_{5}$ described in Example 4.15 can be rewritten respectively as

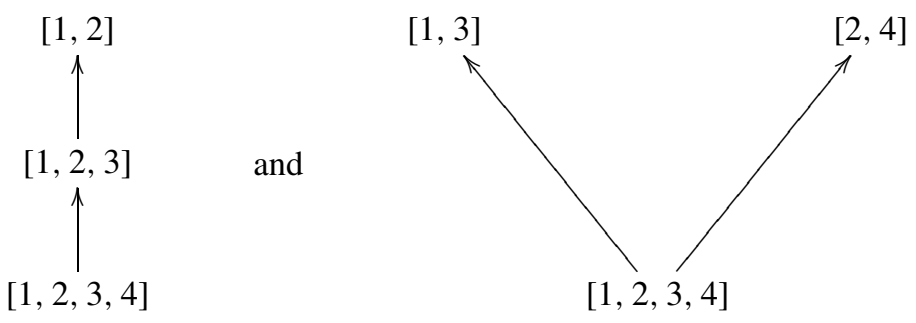


Lemmas 7.3, 8.6, and 10.4 describe the decomposition of $\mathcal{A} \cap H$ in irreducible nested sets and lead to the following result:

Proposition 6.2. Let $T$ be the tree associated to an irreducible classical root system. Then $T$ is a connected tree for which every vertex is adjacent to at most two other vertices.

Lemma 6.3. Let $M=\left\{I_{1}, I_{2}, \ldots, I_{r}\right\}$ be an MPNS. Here we have numbered our irreducible sets such that $\theta\left(I_{1}\right)<\theta\left(I_{2}\right)<\cdots<\theta\left(I_{r}\right)$. Let $k$ be an integer smaller than or equal to $r$. Then the set $\left\{I_{1}, I_{2}, \ldots, I_{k}\right\}$ is saturated.

Indeed, if two sets $I, J$ belong to $M$ and $I \subset J$, then $\theta(I)<\theta(J)$.

Proposition 6.4. Let $M=\left[I_{1}, I_{2}, \ldots, I_{r}\right]$ be a maximal nested proper family. Let $\left[I_{1}^{\prime}, I_{2}^{\prime}, \ldots, I_{r}^{\prime}\right]$ be a reordering of the sequence $\left[I_{1}, I_{2}, \ldots, I_{r}\right]$. We assume that this reordering is compatible with the partial order given by inclusion: if $I_{j}^{\prime} \subset I_{k}^{\prime}$ then $j<k$. Let

$$
\vec{v}=\left[\theta\left(I_{1}\right), \theta\left(I_{2}\right), \ldots, \theta\left(I_{r}\right)\right]
$$

and

$$
\overrightarrow{v^{\prime}}=\left[\theta\left(I_{1}^{\prime}\right), \theta\left(I_{2}^{\prime}\right), \ldots, \theta\left(I_{r}^{\prime}\right)\right]
$$

Then we have $\operatorname{Ires}_{\vec{v}}=\operatorname{Ires}_{\overrightarrow{v^{\prime}}}$.

Proof. We prove this proposition by induction on $r$.

If $\mathcal{A}$ is irreducible, then necessarily $I_{r}=I_{r}^{\prime}=\mathcal{A}$ and $\left[I_{1}^{\prime}, I_{2}^{\prime}, \ldots, I_{r-1}^{\prime}\right]$ is a reordering of the sequence $\left[I_{1}, I_{2}, \ldots, I_{r-1}\right]$. Furthermore, the families $\left\{I_{1}, I_{2}, \ldots, I_{r-1}\right\}$ and $\left\{I_{1}^{\prime}, I_{2}^{\prime}, \ldots, I_{r-1}^{\prime}\right\}$ are MPNSs for $\mathcal{A}_{0}=\bigcup_{j=1}^{r-1} I_{j}$. The set $\mathcal{A}_{0}$ spans a codimension 1 vector space in $V$.

To prove that Ires $\vec{v}=$ Ires $_{\overrightarrow{v^{\prime}}}$, it suffices to test it on basic fractions $f_{\sigma}$. Let $\sigma=$ $\left\{\beta_{1}, \beta_{2}, \ldots, \beta_{r}\right\}$ be a basic subset of $\mathcal{A}$. By Lemma 4.3, if $\operatorname{Ires}_{\vec{v}} f_{\sigma} \neq 0$, then the set $\sigma \cap\left\langle\mathcal{A}_{0}\right\rangle$ is of cardinality $r-1$, and there exists an element of $\sigma$, say $\beta_{r}$, of the form $c \theta+\xi$ where $\xi$ belongs to $\left\langle\mathcal{A}_{0}\right\rangle, c$ is a non-zero constant, and $\theta$ is the highest element of $\mathcal{A}$. Let

$$
\vec{v}_{0}=\left[\theta\left(I_{1}\right), \theta\left(I_{2}\right), \ldots, \theta\left(I_{r-1}\right)\right]
$$

and

$$
\overrightarrow{v_{0}^{\prime}}=\left[\theta\left(I_{1}^{\prime}\right), \theta\left(I_{2}^{\prime}\right), \ldots, \theta\left(I_{r-1}^{\prime}\right)\right]
$$

Then we have

$$
\operatorname{Ires}_{\vec{v}} f_{\sigma}=\frac{1}{c} \operatorname{Ires}_{\overrightarrow{v_{0}}} f_{\sigma \cap\left\langle\mathcal{A}_{0}\right\rangle}
$$

and

$$
\operatorname{Ires}_{\overrightarrow{v^{\prime}}} f_{\sigma}=\frac{1}{c} \operatorname{Ires}_{\overrightarrow{v_{0}^{\prime}}} f_{\sigma \cap\left\langle\mathcal{A}_{0}\right\rangle} \text {. }
$$

We conclude by induction. 
When $\mathcal{A}$ is not irreducible, we write $\mathcal{A}=\bigcup_{a=1}^{s} J_{a}$ where $J_{a}$ are irreducibles. We have $V=\bigoplus_{a=1}^{s}\left\langle J_{a}\right\rangle$. Every basic subset $\sigma$ of $\mathcal{A}$ is the union of basic subsets for the irreducible sets $J_{a}$. Define

$$
\vec{v}_{a}=\left[\theta\left(I_{a}^{i_{1}}\right), \theta\left(I_{a}^{i_{2}}\right), \ldots, \theta\left(J_{a}\right)\right],
$$

where $\left[I_{a}^{i_{1}}, I_{a}^{i_{2}}, \ldots, J_{a}\right]$ is the subsequence of irreducible sets contained in $J_{a}$ extracted (with conserving order) from the sequence $\left[I_{1}, I_{2}, \ldots, I_{r}\right]$. Similarly let

$$
{\overrightarrow{v^{\prime}}}_{a}^{\prime}=\left[\theta\left(I_{a}^{\prime i_{1}}\right), \theta\left(I_{a}^{\prime i_{2}}\right), \ldots, \theta\left(J_{a}\right)\right]
$$

where $\left[I_{a}^{\prime i_{1}}, I_{a}^{\prime i_{2}}, \ldots, I_{a}^{\prime}\right]$ is the subsequence of irreducible sets contained in $J_{a}$ extracted from the sequence $\left[I_{1}^{\prime}, I_{2}^{\prime}, \ldots, I_{r}^{\prime}\right]$. Then, as the calculation takes place with respect to independent variables, we have

$$
\begin{aligned}
\operatorname{Ires}_{\vec{v}}\left(f_{\sigma}\right) & =\prod_{a=1}^{s}\left(\operatorname{Ires}_{\overrightarrow{v_{a}}} f_{\sigma \cap\left\langle J_{a}\right\rangle}\right), \\
\operatorname{Ires}_{\overrightarrow{v^{\prime}}}\left(f_{\sigma}\right) & =\prod_{a=1}^{s}\left(\operatorname{Ires}_{\overrightarrow{v^{\prime}}} f_{\sigma \cap\left\langle J_{a}\right\rangle}\right) .
\end{aligned}
$$

Each of the vector spaces $\left\langle J_{a}\right\rangle$ is of dimension less than $r$, so that by induction hypothesis $\operatorname{Ires}_{\vec{v}_{a}}=\operatorname{Ires}_{\vec{v}_{a}^{\prime}}$. This concludes the proof.

We now consider partial iterated residues. To a set $v$ of elements of $\mathcal{A}$, we associate the vector space

$$
H_{\nu}:=\{u \in U \mid\langle\alpha, u\rangle=0 \text { for all } \alpha \in v\} .
$$

A linear function $\alpha \in \mathcal{A}$ produces a linear function on $H_{v}$ by restriction. If $\vec{v}:=$ $\left[\alpha_{1}, \alpha_{2}, \ldots, \alpha_{k}\right]$ is a sequence of elements of $\mathcal{A}$, the partial iterated residue

$$
\operatorname{Ires}_{\vec{v}} \varphi:=\operatorname{res}_{\alpha_{k}=0} \cdots \operatorname{res}_{\alpha_{1}=0} \varphi
$$

associates to a rational function $\varphi$ in $R_{\mathcal{A}}$ a rational function on $H_{\nu}$ of the form

$$
\frac{G}{\prod_{i=1, \ldots, n ; \overline{\alpha_{i}} \neq 0} \bar{\alpha}_{i}^{n_{i}}},
$$

where $G$ is a polynomial function on $H_{v}$ and $\bar{\alpha}$ is the restriction of $\alpha$ to $H_{v}$. Let $M$ be an MPNS and consider the tree associated to $M$. Given a saturated subset $S$ of $M$, we can define the iterated residue with respect to this saturated set: we choose any order $S:=\left[I_{1}, I_{2}, \ldots, I_{k}\right]$ on $S$ compatible with the inclusion relation and define Ires ${ }_{S}:=$ Ires $_{\vec{v}}$ with $\vec{v}=\left[\theta\left(I_{1}\right), \theta\left(I_{2}\right), \ldots, \theta\left(I_{k}\right)\right]$. With the same proof as for Proposition 6.4, this partial residue depends only on the set $S$. We denote by $H_{S}$ the intersection of the kernels of the elements $\alpha$ for $\alpha \in S$. It is also the intersection of the kernels of the elements $\theta\left(I_{k}\right)$, as the set $v$ is a basic sequence in $S$.

Let $\varphi$ be a function in $R_{\mathcal{A}}$ of the form

$$
\varphi=\frac{P}{\prod_{i=1}^{n} \alpha_{i}} .
$$


Let $M$ be an MPNS and let $J_{1}, J_{2}, \ldots, J_{s}$ be elements of $M$. We consider the saturated subset $S$ of $M$ consisting of the elements of the tree strictly above $J_{1}, J_{2}, \ldots, J_{s}$. The iterated residue $\operatorname{Ires}_{S} \varphi$ is a function on $H_{S}$. Denote by $u_{a}$ the restriction of the function $\theta\left(J_{a}\right)$ to $H_{S}$.

Proposition 6.5. The pole of the linear function $u_{a}$ in the iterated residue $\operatorname{Ires}_{S} \varphi$ is of order less than or equal to $\left|J_{a}\right|-\operatorname{dim}\left\langle J_{a}\right\rangle+1$.

See Figs. 7 and 8 for an application of the proposition.

Proof. Choose a vector space $E$ such that

$$
V=\left\langle J_{1}\right\rangle \oplus \cdots \oplus\left\langle J_{s}\right\rangle \oplus E .
$$

Let $B=\bigcup_{a=1}^{s} J_{a}$ and $C=\mathcal{A} \backslash B$. Write $C:=\left\{\beta_{1}, \beta_{2}, \ldots, \beta_{q}\right\}$ and

$$
\varphi=P \times \varphi_{1} \times \varphi_{2} \times \cdots \times \varphi_{s} \times Q
$$

with $\varphi_{a}=1 / \prod_{\alpha \in J_{a}} \alpha$ and $Q=1 / \prod_{j=1}^{q} \beta_{j}$.

For $\beta_{j} \in C$, we write $\beta_{j}=\sum_{i=1}^{s} \beta_{j}^{i}+\gamma_{j}$ with $\beta_{j}^{i} \in\left\langle J_{i}\right\rangle$ and $\gamma_{j} \in E$. The element $\gamma_{j}$ is necessarily non-zero, as the set $B$ is complete. Thus we write

$$
\frac{1}{\beta_{j}}=\frac{1}{\gamma_{j}\left(1+\left(\sum_{i=1}^{s} \beta_{j}^{i}\right) / \gamma_{j}\right)}
$$

and the iterated residue is by definition

$$
\operatorname{Ires}_{S}(\varphi)=\operatorname{Ires}_{S}\left(P \times\left(\varphi_{1} \cdots \varphi_{s}\right) \times \prod_{j=1}^{q} \frac{1}{\gamma_{j}} \sum_{k=0}^{\infty}\left((-1)^{k} \frac{\sum_{i=1}^{s} \beta_{j}^{i}}{\gamma_{j}}\right)^{k}\right) .
$$

Here, when taking the residue, the elements $\gamma_{j}$ are considered as constants and this sum is finite.

Consider the subset $M_{a}$ of elements of $M$ contained in $J_{a}$. This is an MPNS for the set $J_{a}$. Let $J_{a}^{+}$be the saturated subset of $M_{a}$ consisting of all elements of $M_{a}$ different from $J_{a}$. Then $J_{a}^{+}$has $\operatorname{dim}\left\langle J_{a}\right\rangle-1$ elements. If $g=P_{a} / \prod_{\alpha \in J_{a}} \alpha^{n_{\alpha}}$, the iterated residue Ires $_{J_{a}^{+}} g$ is a Laurent polynomial in $u_{a}$.

Now $\operatorname{Ires}_{S} \varphi$ is a sum of products of residues of the form $\operatorname{Ires}_{J_{a}^{+}} g_{a}$ where $g_{a}=$ $P_{a} / \prod_{\alpha \in J_{a}} \alpha$ and $P_{a}$ is a polynomial. Thus we obtain a Laurent polynomial in $u_{a}, a=$ $1, \ldots, s$ (with coefficients rational functions on the vector space $E^{*}$ ). Now the homogeneous degree of $g_{a}$ is greater than or equal to $-\left|J_{a}\right|$. The number of residues we are taking is equal to $\operatorname{dim}\left\langle J_{a}\right\rangle-1$. So we obtain a function of $u_{a}$ of homogeneous degree greater than or equal to $-\left|J_{a}\right|+\operatorname{dim}\left\langle J_{a}\right\rangle-1$. This means that the pole in $u_{a}$ is of order less than or equal to $\left|J_{a}\right|-\operatorname{dim}\left\langle J_{a}\right\rangle+1$.

Let us consider the MPNS whose tree representation is given by Fig. 7. The orders of the poles of its nodes are given in Fig. 8. 


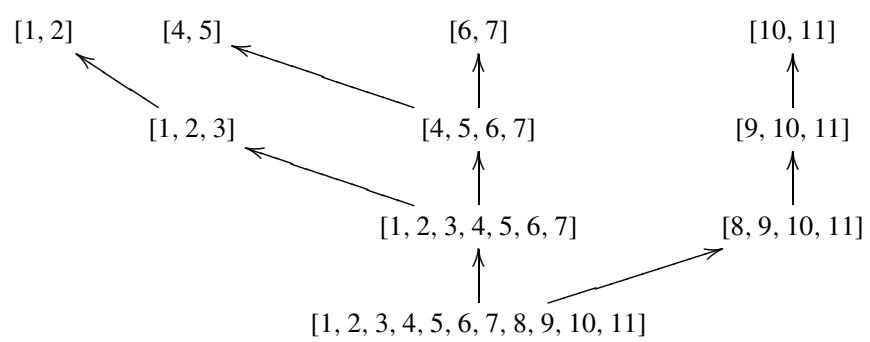

Fig. 7. Irreducible components of an MPNS in $A_{10}$.

Remark 6.6. In our program for calculating iterated residues for root systems of type $A_{r}$, we reorder roots according to the tree order: we take the residue first with respect to the elements $\theta\left(I_{k}\right)$ appearing at the end of the tree in arbitrary order, and we remove these variables. Then we take the variables appearing at the end of the tree when we have removed these irreducible sets. Here an irreducible set $I$ is indexed by a subset $S$ of $\{1,2, \ldots, r+1\}$. A subset $S$ of cardinality 2 , for example [1, 3], corresponds to the irreducible set with one element (here $e_{1}-e_{3}$ ). Thus given an MNS $M$ represented as $M=\left\{S_{1}, S_{2}, \ldots, S_{r}\right\}$ we first take the residues with respect to the roots $\theta\left(I_{k}\right)$, for sets $S_{k}$ of cardinality 2 , in arbitrary order, then with respect to irreducible sets associated to sets $S_{k}$ of cardinality 3 , etc. The procedure of ordering roots coming from an MNS $M=\left\{S_{j}\right\}$ according to the cardinality of the set $S_{k}$ is called OrderThetas. Furthermore, at the same time we keep track of the order of the pole for calculating an iterated residue of a function $\varphi=P / \prod_{i=1}^{N} \alpha_{i}$ in the procedure FormalPathAwithorders.

\section{Volume and Partition Function for the System $A_{n-1}$}

\subsection{The Formulas to Be Implemented}

Let $E$ be an $n$-dimensional vector space with basis $e_{i}(i=1, \ldots, n)$ and consider the set

$$
\mathcal{K}_{n}=\left\{e_{i}-e_{j} \mid 1 \leq i<j \leq n\right\}
$$

These are the positive roots for a system of type $A_{n-1}$. The number of elements in $\mathcal{K}_{n}$ is $N=n(n-1) / 2$. Note that $\mathcal{K}_{n}$ is also the set of vectors in a complete graph with $n$ nodes.

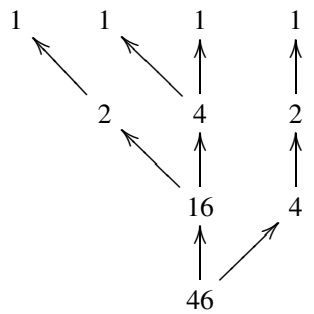

Fig. 8. Order of nodes in the tree represented in Fig. 7, according to Proposition 6.5. 
We let $V$ be the vector space generated by the elements in $\mathcal{K}_{n}$. Then $V$ has dimension $n-1$ and it is defined by

$$
V=\left\{v=\sum_{i=1}^{n} v_{i} e_{i} \in E \mid \sum_{i=1}^{n} v_{i}=0\right\} .
$$

In our procedures, a vector $v$ of length $n$ such that $\sum_{i=1}^{n} v_{i}=0$ is called an A-vector and written as $v=\left[v_{1}, v_{2}, \ldots, v_{n}\right]$. The lattice spanned by $\mathcal{K}_{n}$ is simply

$$
V_{\mathbb{Z}}=\left\{h=\sum_{i=1}^{n} h_{i} e_{i} \in \mathbb{Z}^{n} \mid \sum_{i=1}^{n} h_{i}=0\right\} .
$$

It is well known and easy to prove that $\mathcal{K}_{n}$ is unimodular. The cone $\mathcal{C}\left(\mathcal{K}_{n}\right)$ generated by $\mathcal{K}_{n}$ is simplicial with generators the $n-1$ simple roots $e_{1}-e_{2}, e_{2}-e_{3}, \ldots, e_{n-1}-e_{n}$. This cone is described as

$$
\mathcal{C}\left(\mathcal{K}_{n}\right)=\left\{A \text {-vector } v=\left[v_{1}, v_{2}, \ldots, v_{n}\right] \mid v_{1}+v_{2}+\cdots+v_{i} \geq 0 \text { for all } i\right\} .
$$

Keep in mind that our vector $v$ satisfies the condition

$$
v_{1}+v_{2}+\cdots+v_{n-1}+v_{n}=0 .
$$

We choose on $V$ the measure $d h$ determined by $V_{\mathbb{Z}}$. Let $v$ be in the cone $\mathcal{C}\left(\mathcal{K}_{n}\right)$. We are interested in computing the volume $\operatorname{vol}_{\mathbb{Z}, \mathcal{K}_{n}}(v)$ of the polytope

$$
\Pi_{\mathcal{K}_{n}}(v)=\left\{\left(x_{\alpha}\right)_{\alpha} \in \mathbb{R}^{N} \mid x \geq 0, \sum_{\alpha \in \mathcal{K}_{n}} x_{\alpha} \alpha=v\right\} .
$$

If $h$ is a point in $V$ with integral coordinates then we are also interested in computing the number $N_{\mathcal{K}_{n}}(h)$ of integral points in $\Pi_{\mathcal{K}_{n}}(h)$.

We apply the formulas of Theorem 3.3. Since $\mathcal{K}_{n}$ is unimodular, the set $\Gamma$ can be taken as $\Gamma:=\{0\}$ (Remark 3.9).

Since $V$ is contained in $E$, then we have a canonical map $E^{*} \longrightarrow V^{*}$ given by restriction. Define $U=V^{*}$ as in the general setting. We identify $U$ with $\mathbb{R}^{n-1}$ by sending $u \in \mathbb{R}^{n-1}$ to $u=\sum_{i=1}^{n-1} u_{i} e^{i} \in E^{*}$, where $e^{i}$ is the dual basis to $e_{i}$. Thus the root $e_{i}-e_{j}(1 \leq i<j<n)$ produces the linear function $u_{i}-u_{j}$ on $U$, while the root $e_{i}-e_{n}$ produces the linear function $u_{i}$.

Definition 7.1. Let $v=\sum_{i=1}^{n} v_{i} e_{i} \in V$ be a vector with real coordinates. Let $h=$ $\sum_{i=1}^{n} h_{i} e_{i} \in V$ be a vector with integral coordinates. Then for $u \in U$ define:

$$
\begin{aligned}
& \text { - } J_{A}(v)(u)=e^{\sum_{i=1}^{n-1} u_{i} v_{i}} / \prod_{i=1}^{n-1} u_{i} \prod_{1 \leq i<j \leq n-1}\left(u_{i}-u_{j}\right) . \\
& \text { - } \mathcal{F}_{A}(h)(u)=\prod_{i=1}^{n-1}\left(1+u_{i}\right)^{h_{i}+n-1-i} / \prod_{i=1}^{n-1} u_{i} \prod_{1 \leq i<j \leq n-1}\left(u_{i}-u_{j}\right) .
\end{aligned}
$$


Theorem 7.2. Let $\mathfrak{c}$ be a chamber of $\mathcal{C}\left(\mathcal{K}_{n}\right)$.

- For $v \in \overline{\mathfrak{c}}$, we have

$$
\operatorname{vol}_{\mathbb{Z}, \mathcal{K}_{n}}(v)=\mathrm{JK}_{\mathfrak{c}}\left(J_{A}(v)\right) .
$$

- For $h \in \mathbb{Z}^{n} \cap \overline{\mathfrak{c}}$, we have

$$
N_{\mathcal{K}_{n}}(h)=\mathrm{JK}_{\mathfrak{c}}\left(\mathcal{F}_{A}(h)\right) .
$$

Proof. The first assertion is the general formula.

The function $F(0, h)(u)=e^{\langle h, u\rangle} / \prod_{\alpha \in \mathcal{A}}\left(1-e^{-\langle\alpha, u\rangle}\right)$ for the system $\mathcal{K}_{n}$ is

$$
F(0, h)(u)=\frac{e^{\sum_{i=1}^{n-1} u_{i} v_{i}}}{\prod_{i=1}^{n-1}\left(1-e^{-u_{i}}\right) \prod_{1 \leq i<j \leq n-1}\left(1-e^{-\left(u_{i}-u_{j}\right)}\right)} .
$$

Note that the change of variable $1+z_{i}=e^{u_{i}}$ preserves the hyperplanes $u_{i}=0$ and $u_{i}=u_{j}$. After the change of variable, we get

$$
F(0, h)(u)=\frac{\prod_{i=1}^{n-1}\left(1+z_{i}\right)^{h_{i}+n-i}}{\prod_{1 \leq i<j \leq n-1}\left(z_{i}-z_{j}\right) \times \prod_{i=1}^{n-1} z_{i}} .
$$

However, $z_{i}=e^{u_{i}}-1$ leads to $d z_{i}=e^{u_{i}} d u_{i}=\left(1+z_{i}\right) d u_{i}$ and hence we obtain the desired exponent $h_{i}+n-i-1$ thanks to the formula involving Jacobians in Proposition 3.2.

In order to implement these formulas, we first have to describe the set $\mathcal{P}\left(v, \mathcal{K}_{n}\right)$ (Section 7.2), then calculate the iterated residue formulas associated to these paths (Section 7.3), and then add them. We stress the fact that our programs work with formal parameters.

\subsection{The Search for Maximal Proper Nested Sets Adapted to a Vector}

We now look for MPNSs adapted to a vector following the general method as outlined in Fig. 6: we begin by listing all possible $\mathcal{K}_{n}$-admissible hyperplanes. The usual height function is

$$
\mathrm{ht}(v)=\sum_{i=1}^{n-1}(n-i) v_{i}
$$

which takes the value 1 on all the simple roots, and hence the value $j-i$ on $e_{i}-e_{j}$. We deform ht slightly in order to have a function taking different values on all roots: If two elements $e_{i}-e_{j}$ and $e_{k}-e_{\ell}$ are such that $j-i=\ell-k$, we decide that $\operatorname{ht}\left(e_{i}-e_{j}\right)<\operatorname{ht}\left(e_{k}-e_{\ell}\right)$ if $i<k$.

If $P$ is a proper subset of $\{1,2, \ldots, n\}$ and $v$ is an $A$-vector, we denote by $\left\langle u_{P}, v\right\rangle$ the linear form $\sum_{i \in P} v_{i}$, and by $H_{P}$ the hyperplane

$$
H_{P}:=\left\{v \in V \mid\left\langle u_{P}, v\right\rangle=0\right\} .
$$


Observe that the hyperplane $H_{P}$ is equal to the hyperplane $H_{Q}$ determined by the complement $Q$ of $P$. We denote

$$
\mathcal{K}(P):=\left\{e_{i}-e_{j} \mid 1 \leq i<j \leq n ; i, j \in P\right\} \subset \mathcal{K}_{n} .
$$

Note that $\mathcal{K}(P)$ is the positive system $A_{P \mid-1}$, where the positivity is induced by the lexicographic order.

\section{Lemma 7.3.}

- The hyperplane $H_{P}$ is a $\mathcal{K}_{n}$-admissible hyperplane and every $\mathcal{K}_{n}$-admissible hyperplane is of this form.

- The set $\mathcal{K}_{n} \cap H_{P}$ is the union of $\mathcal{K}(P)$ and $\mathcal{K}(Q)$, where $Q$ is the complement of $P$ in $\{1,2, \ldots, n\}$.

Proof. Let $H$ be a $\mathcal{K}_{n}$-admissible hyperplane. Let us prove that $H=H_{P}$ for some proper subset $P$ of $\{1,2, \ldots, n\}$ by induction on $n$. Let $\alpha$ be a root in $H$. Renumbering the roots, we may assume that $\alpha=e_{n-1}-e_{n}$. The map $q$ sending $e_{i}$ to $e_{i}$ if $i<n$ and $e_{n}$ to $e_{n-1}$ sends the set $\mathcal{K}_{n} \backslash\{\alpha\}$ to $\mathcal{K}_{n-1}$. The space $H / \mathbb{R} \alpha$ becomes a $\mathcal{K}_{n-1}$-admissible hyperplane. It is thus determined by a subset $P^{\prime}$ of $\{1,2, \ldots, n-1\}$. If $P^{\prime}$ does not contain $n-1$, the hyperplane $H$ is equal to the hyperplane determined by the subset $P^{\prime}$ of $\{1,2, \ldots, n-1\}$. If $P^{\prime}$ does contain $n-1$, then the hyperplane $H$ is equal to the hyperplane determined by $P=P^{\prime} \cup\{n\}$. The other assertions of the lemma are easy to see.

We now proceed to the description of our algorithm. Recall our description of an $A$-vector as an array $v=\left[v_{1}, v_{2}, \ldots, v_{n}\right]$ with $\sum_{i=1}^{n} v_{i}=0$. Referring to Fig. 6 we need to check if the vector is in the cone $\mathcal{C}\left(\mathcal{K}_{n}\right)$, that is, $\sum_{j=1}^{i} v_{j} \geq 0$ for $1 \leq i \leq n-1$. This is done by using the procedure CheckVector $(\mathrm{v})$, which gives an answer true or false.

For the system $\mathcal{K}_{n}$ the highest $\operatorname{root} \theta$ is equal to

$$
\theta=[1,0, \ldots, 0,-1] \in \mathbb{R}^{n} .
$$

We list all hyperplanes that are in $\operatorname{Hyp}\left(v, \mathcal{K}_{n}\right)$. This is done in the procedure TwoSets(v), that we are about to describe. As explained in Lemma 7.3, each hyperplane is determined by an equation $\sum_{i \in I} a_{i}=0$. It therefore produces a set of two lists $P, Q$, where $P=[i \in I]$ and $Q=[i \notin I \mid 1 \leq i \leq n]$. Note that $P$ and $Q$ are sorted. To verify that such a hyperplane is in $\operatorname{Hyp}\left(v, \mathcal{K}_{n}\right)$, we need to test if $\left\langle u_{P}, \theta\right\rangle$ is not zero ( $\theta$ is not in the hyperplane) and if $\left\langle u_{P}, v\right\rangle \times\left\langle u_{P}, \theta\right\rangle$ is non-negative ( $\theta$ and $v$ are on the same side of the hyperplane). Furthermore, the procedure ProjH(v, H) constructs the vector

$$
\operatorname{proj}_{H}(v)=v-\frac{\left\langle u_{P}, v\right\rangle}{\left\langle u_{P}, \theta\right\rangle} \theta
$$

that we represent as $\left\{\left[v_{1}, P\right],\left[v_{2}, Q\right]\right\}$. Each of the vectors $v_{1}, v_{2}$ is an $A$-vector (sum of coordinates equal to zero). So the last condition for $H$ being in $\operatorname{Hyp}\left(v, \mathcal{K}_{n}\right)$ is that $v_{1} \in \mathcal{C}(\mathcal{K}(P))$ and $v_{2} \in \mathcal{C}(\mathcal{K}(Q))$. 
Hence a hyperplane $H_{P}$ is in $\operatorname{Hyp}\left(v, \mathcal{K}_{n}\right)$ if it satisfies the series of conditions:

$$
\begin{aligned}
& \left\langle u_{P}, \theta\right\rangle \neq 0 \quad \text { with } \operatorname{Hvalue}(\operatorname{theta}(\mathrm{n}), \mathrm{P}) \neq 0 \text {, } \\
& \left\langle u_{P}, v\right\rangle \times\left\langle u_{P}, \theta\right\rangle \geq 0 \quad \text { with } \quad \operatorname{CheckSide}(\mathrm{v}, \mathrm{P})=\text { true, } \\
& v_{1} \in \mathcal{C}\left(\mathcal{K}_{|P|-1}\right) \quad \text { with } \quad \text { CheckVector }\left(\mathrm{v}_{1}\right)=\text { true, } \\
& v_{2} \in \mathcal{C}\left(\mathcal{K}_{|Q|-1}\right) \quad \text { with } \quad \text { CheckVector }\left(\mathrm{v}_{2}\right)=\text { true. }
\end{aligned}
$$

If all answers are true, then $H_{P}$ is in $\operatorname{Hyp}\left(v, \mathcal{K}_{n}\right)$ and we obtain two vectors, [ $\left.v_{1}, P\right]$, $\left[v_{2}, Q\right]$, from our input $(v, P)$. Then we construct the MNSs for $\left[v_{1}, P\right]$ and $\left[v_{2}, Q\right]$, and go on recursively until the procedure stops. These iterated steps are done by the procedure Splits. Finally the procedure MNSs(v) computes the set $\mathcal{P}\left(v, \mathcal{K}_{n}\right)$ for a given vector $v$, by running over all subsets $P$ such that $H_{P}$ is in $\operatorname{Hyp}\left(v, \mathcal{K}_{n}\right)$.

\subsection{Residues Associated to Maximal Proper Nested Sets}

An element $M$ in $\mathcal{P}\left(v, \mathcal{K}_{n}\right)$ is represented as a collection $M=\left\{K_{1}, K_{2}, \ldots, K_{n-1}\right\}$ of $(n-1)$ subsets of $[1,2, \ldots, n]$. As we have said in Remark 6.6, given an MPNS

$M:=\left\{K_{1}, K_{2}, \ldots, K_{n-1}\right\}$ we associate to it an ordered basis $\overrightarrow{\theta(M)}$ of $V$ (procedure OrderThetas). If $p=\left[\alpha_{1}, \alpha_{2}, \ldots, \alpha_{n-1}\right]$ is the list of roots singled out by our procedure, then $\alpha_{1}$ is an element associated to a set $K_{i}$ of cardinality 2 and $\alpha_{n-1}=\theta$. We identify the root $e_{i}-e_{n}$ to the linear function $z_{i}$ on $\mathbb{C}^{n-1}$ and the root $e_{i}-e_{j}$ to $z_{i}-z_{j}$.

Let $h$ be an $A$-vector with integral coordinates. Let us consider the Kostant function (Definition 7.1)

$$
\mathcal{F}_{A}(h)\left(z_{1}, z_{2}, \ldots, z_{n-1}\right)=\frac{\prod_{i=1}^{n-1}\left(1+z_{i}\right)^{h_{i}+n-1-i}}{\prod_{1 \leq i<j \leq n-1}\left(z_{i}-z_{j}\right) \times \prod_{i=1}^{n-1} z_{i}} .
$$

To compute $N_{\mathcal{K}_{n}}(h)$, we have to compute

$$
\operatorname{res}_{M} \varphi:=\operatorname{res}_{\alpha_{n-1}=0} \operatorname{res}_{\alpha_{2}=0} \cdots \operatorname{res}_{\alpha_{1}=0} \varphi
$$

with $\varphi=\mathcal{F}_{A}(h)$. Our procedure works either if we assign some numerical value to $h$, or if $h$ is a symbolic parameter. In this section we explain how to compute the polynomial $h \rightarrow N_{\mathcal{K}_{n}}(h)$.

If $\alpha_{1}=z_{i}-z_{j}$, we can replace-after taking the residue at $z_{i}=z_{j}$-the variable $z_{i}$ by the variable $z_{j}$ in all the other roots. Thus we eliminate the variable $z_{i}$. Recursively, we have to compute the residue at $z_{i_{0}}-z_{j_{0}}=0$ of an expression

$$
f=\frac{A\left(z_{i}, h, i \in L\right) \prod_{i \in L}\left(1+z_{i}\right)^{h_{i}+n-1-i}}{\prod_{i, j \in L ; i<j}\left(z_{i}-z_{j}\right)^{m_{i, j}} \prod_{i \in L} z_{i}^{m_{i}}},
$$

where $L$ is a list of indices taken in $\{1, \ldots, n-1\}$, and $A\left(z_{i}, h\right)$ is a polynomial in $z_{i}$ and $h$. Recall that using Proposition 6.5, we know in advance the order maxi $=m_{i_{0}, j_{0}}$ of the root $z_{i_{0}}-z_{j_{0}}$. Note that computing the residue is exactly the same as computing the coefficient of $z$ of degree maxi -1 of the expansion of $f \times\left(z_{i_{0}}-z_{j_{0}}\right)^{\text {maxi }}$ at $z_{i_{0}}=z+z_{j_{0}}$. 
For any parameter $b$, we write at $z_{i_{0}}=z+z_{j_{0}}$,

$$
\left(1+z_{i_{0}}\right)^{b}=\left(1+z_{j_{0}}\right)^{b}\left(1+\sum_{j=1}^{\operatorname{maxi}-1}\left(\begin{array}{l}
b \\
j
\end{array}\right)\left(\frac{z}{1+z_{j_{0}}}\right)^{j}\right)+O\left(z^{\operatorname{maxi}}\right) .
$$

Similarly, we write at $z_{i_{0}}=z+z_{j_{0}}$,

$$
\frac{1}{\left(z_{i}-z_{i_{0}}\right)^{m_{i, i_{0}}}}=\frac{1}{\left(z_{i}-z_{j_{0}}\right)^{m_{i, i_{0}}}}\left(1-\frac{z}{z_{i}-z_{j_{0}}}\right)^{-m_{i, i_{0}}}
$$

and we expand it as a series in $z$ in the procedure CoeffBin using the binomial coefficients.

Finally after expanding all factors of $f$ containing the variable $z_{i_{0}}=z+z_{j_{0}}$ in the variable $z$, we compute the coefficient of $z^{\operatorname{maxi-1}}$.

\subsection{The Procedure MNS_KostantA}

We finish the section dedicated to $A_{n-1}$ by giving the global outline of the procedure MNS_KostantA $(\mathrm{v})$ computing the Kostant partition number of a vector $v$ lying in the root lattice. We begin by slightly deforming $v$ so that it lies on no admissible hyperplanes, with the command $\mathrm{v}^{\prime}:=\operatorname{DefVector}(\mathrm{v}, \mathrm{n})$. We compute all MPNSs for $v^{\prime}$ with the procedure $\operatorname{MNSS}\left(\mathrm{v}^{\prime}\right)$.

Given such an MPNS $M=\left\{S_{k}\right\}$, we extract the highest roots of its irreducible components with the call $\mathrm{R}:=$ ThetaMNS(M). We obtain a set $R$ where each element of $R$ is a root represented as $[i, j]$ together with the cardinality of the set $S_{k}$ it comes from. We then transform this set $R$ into a path $p$ keeping track of the order of poles by setting $\mathrm{p}:=$ FormalPathAwithorders(R).

Finally we compute the residue associated to this path with OneIteratedResidue(p,v, n). Summing all these residues over the set of MNSs, we obtain, thanks to Theorem 7.2, the desired partition number for $v$.

\section{Type $B_{n}$}

\subsection{The Formulas to Be Implemented}

Consider a vector space $V$ with basis $e_{1}, e_{2}, \ldots, e_{n}$. We choose on $V$ the standard Lebesgue measure $d h$. Let

$$
\mathcal{B}_{n}=\left\{e_{i} \mid 1 \leq i \leq n\right\} \cup\left\{e_{i}-e_{j} \mid 1 \leq i<j \leq n\right\} \cup\left\{e_{i}+e_{j} \mid 1 \leq i<j \leq n\right\} .
$$

Then $\mathcal{B}_{n}$ is a positive roots system of type $B_{n}$ and generates $V$. The number of elements in $\mathcal{B}_{n}$ is $N=n^{2}$. We denote by $U$ the dual of $V$. The lattice $V_{\mathbb{Z}}$ generated by roots is equal to $\mathbb{Z}^{n}$, so the constant vol $\left(V / V_{\mathbb{Z}}, d h\right)=1$.

The cone $\mathcal{C}\left(\mathcal{B}_{n}\right)$ is simplicial and spanned by the $n$ simple roots $e_{1}-e_{2}, e_{2}-e_{3}, \ldots$, $e_{n-1}-e_{n}, e_{n}$. A vector $v=\left[v_{1}, v_{2}, \ldots, v_{n}\right]$ is in $\mathcal{C}\left(\mathcal{B}_{n}\right)$ if and only if it satisfies the inequalities $v_{1}+\cdots+v_{i} \geq 0$ for all $i=1, \ldots, n$. 
Let $v$ be in the cone $\mathcal{C}\left(\mathcal{B}_{n}\right)$. Consider the polytope

$$
\Pi_{\mathcal{B}_{n}}(v)=\left\{\left(x_{\alpha}\right)_{\alpha} \geq 0 \mid \sum_{\alpha \in \mathcal{B}_{n}} x_{\alpha} \alpha=v\right\} .
$$

If $h$ is a point in $V$ with integral coordinates, we are interested in computing the number $N_{\mathcal{B}_{n}}(h)$ of integral points in $\Pi_{\mathcal{B}_{n}}(h)$.

Let $U_{\mathbb{Z}}$ be the lattice dual to $V_{\mathbb{Z}}$. We identify the torus $T=U / U_{\mathbb{Z}}=\mathbb{R}^{n} / \mathbb{Z}^{n}$ to $\left(S^{1}\right)^{n}$ by

$$
\left(u_{1}, u_{2}, \ldots, u_{n}\right) \mapsto\left(e^{2 \pi \sqrt{-1} u_{1}}, \ldots, e^{2 \pi \sqrt{-1} u_{n}}\right) .
$$

If $G$ is a representative of $g=\left(g_{1}, g_{2}, \ldots, g_{n}\right) \in T$, and $h=\sum_{i=1}^{n} h_{i} e_{i}$ in $V_{\mathbb{Z}}$, then $e^{\langle h, 2 \pi \sqrt{-1} G\rangle}$ is equal to $\prod_{i=1}^{n} g_{i}^{h_{i}}=g^{h}$. As the set $\mathcal{B}_{n}$ is not unimodular, the sets $T(\sigma)$ are not reduced to 1 . Note that we are now using multiplicative notation for $T$, so that we denote the identity by $g=1$. We do the same in the case of $C_{n}$ and $D_{n}$.

Example 8.1. Let $\sigma$ be the basic set $\left\{e_{1}+e_{2}, e_{1}-e_{2}\right\}$ for $B_{2}$. Then $T(\sigma)=\{(1,1)$, $(-1,-1)\}$.

We now determine a set $\Gamma$ containing all sets $T(\sigma)$.

Lemma 8.2. Let $\sigma$ be a basic subset of $\mathcal{B}_{n}$. Assume $g \in T(\sigma)$. Then all the coordinates of $g$ are equal to \pm 1 . Furthermore, if $g$ is not 1 , there are at least two coordinates of $g$ which are equal to -1 .

Proof. We prove this by induction on $n$. For $\mathcal{B}_{2}$, we have seen this by direct computation.

Let $\sigma$ be a basic subset of $\mathcal{B}_{n}$. Assume first that $\sigma$ contains a root $e_{i}$. Up to renumbering, we may assume that this root is $e_{n}$. Then the basis $\sigma$ produces a basis $\sigma^{\prime}$ of $\mathcal{B}_{n-1}$ by putting $e_{n}=0$. Let $g=\left(g_{1}, g_{2}, \ldots, g_{n}\right)$ in $T(\sigma)$. We see that $g^{\prime}=\left(g_{1}, g_{2}, \ldots, g_{n-1}\right)$ is in $T\left(\sigma^{\prime}\right)$. Thus, by induction the first $n-1$ coordinates of $g^{\prime}$ are equal to \pm 1 . However, since $e_{n}$ is in $\sigma$ we get $1=g_{n}$. Note that $g \neq 1$ if and only if $g^{\prime} \neq 1$, hence by induction hypothesis $g^{\prime}$ has at least two coordinates not equal to 1 .

Consider now the case where $\sigma$ does not contain any root $e_{i}$. Up to renumbering, it contains a root $e_{n-1}-e_{n}$ or $e_{n-1}+e_{n}$.

We examine first the case where $\sigma$ contains the root $\alpha=e_{n-1}-e_{n}$. Let $g=$ $\left(g_{1}, g_{2}, \ldots, g_{n-1}, g_{n}\right)$ in $T(\sigma)$. This implies $g_{n-1}=g_{n}$. Consider the map $q$ sending $e_{i}$ to $e_{i}$ if $i<n$ and $e_{n}$ to $e_{n-1}$. Then $q$ sends $\sigma \backslash\left\{e_{n-1}-e_{n}\right\}$ to a basis $\sigma^{\prime}$ of $\mathcal{B}_{n-1}$. The element $g^{\prime}=\left(g_{1}, g_{2}, \ldots, g_{n-1}\right)$ is easily seen to belong to $T\left(\sigma^{\prime}\right)$. Indeed, if $\alpha$ equals $e_{i} \pm e_{j}$ with $1 \leq i<j<n$, this is by definition. On the other hand, $q\left(e_{i} \pm e_{n}\right)=e_{i} \pm e_{n-1}$ and $g_{n-1}=g_{n}$ imply that $g_{i} g_{n-1}^{ \pm 1}$ coincides with the value of $g_{i} g_{n}^{ \pm 1}$. By induction hypothesis, all coordinates of $g^{\prime}$ are equal to \pm 1 . Moreover, $g \neq 1$ if and only if $g^{\prime} \neq 1$, so that $g$ is of the desired form.

Finally, the same argument works if $\sigma$ contains $\alpha=e_{n-1}+e_{n}$, by considering the map $q$ sending $e_{i}$ to $e_{i}$ if $i<n$, and $e_{n}$ to $-e_{n-1}$. 
Definition 8.3. If $I$ is a subset of $\{1,2, \ldots, n\}$ with at least two elements, we consider the set $\Gamma(I):=\left\{\left(g_{1}, g_{2}, \ldots, g_{n}\right) \mid g_{i}=-1, i \in I ; g_{j}=1, j \notin I\right\}$. We define $\Gamma \subset T$ to be the finite subset of $T$ union of such sets $\Gamma(I)$ together with the identity $(1,1, \ldots, 1)$.

Let $v=\sum_{i=1}^{n} v_{i} e_{i} \in V$ be a vector with real coordinates and let $h=\sum_{i=1}^{n} h_{i} e_{i} \in V$ be a vector with integral coordinates. We compute the normalized volume of $\Pi_{\mathcal{B}_{n}}(v)$ and the number of integral points in $\Pi_{\mathcal{B}_{n}}(h)$ using Theorem 3.3. Thus we introduce the function $J_{B}(v)$ on $U$ defined by

$$
J_{B}(v)(u)=\frac{e^{\sum_{i=1}^{n} u_{i} v_{i}}}{\prod_{i=1}^{n} u_{i} \prod_{1 \leq i<j \leq n}\left(u_{i}-u_{j}\right) \prod_{1 \leq i<j \leq n}\left(u_{i}+u_{j}\right)} .
$$

For $g=\left(g_{1}, g_{2}, \ldots, g_{n}\right) \in \Gamma$ and $h \in V_{\mathbb{Z}} \cap \mathcal{C}\left(\mathcal{B}_{n}\right)$ the Kostant fraction (3) is the function on $U$ defined by

$$
\begin{aligned}
F_{B}(g, h)(u)= & \frac{\prod_{i=1}^{n} g_{i}^{h_{i}} e^{\sum_{i=1}^{n} u_{i} h_{i}}}{\prod_{i=1}^{n}\left(1-g_{i}^{-1} e^{-u_{i}}\right) \times \prod_{1 \leq i<j \leq n}\left(1-g_{i}^{-1} g_{j} e^{-\left(u_{i}-u_{j}\right)}\right)} \\
& \times \frac{1}{\prod_{1 \leq i<j \leq n}\left(1-g_{i}^{-1} g_{j}^{-1} e^{-\left(u_{i}+u_{j}\right)}\right)} .
\end{aligned}
$$

We then have

Theorem 8.4. Let $\mathfrak{c}$ be a chamber of $\mathcal{C}\left(\mathcal{B}_{n}\right)$.

- For any $v \in \overline{\mathfrak{c}}$, we have

$$
\operatorname{vol}_{\mathbb{Z}, \mathcal{B}_{n}}(v)=\mathrm{JK}_{\mathfrak{c}}\left(J_{B}(v)\right) .
$$

- For any $h \in V_{\mathbb{Z}} \cap \overline{\mathfrak{c}}$, the value of the partition function is given by

$$
N_{\mathcal{B}_{n}}(h)=\sum_{g \in \Gamma} \mathrm{JK}_{\mathfrak{c}}\left(F_{B}(g, h)\right) .
$$

As in the case of $A_{n}$, we use the change of variable $1+z_{i}=e^{u_{i}}$ to compute $N_{\mathcal{B}_{n}}(h)$ more easily. However, we note that this transformation does not leave the hyperplane $u_{i}+u_{j}=0$ fixed. This hypersurface is transformed into the hypersurface $z_{i}+z_{j}+$ $z_{i} z_{j}=0$. So we use the expression of $\mathrm{JK}_{\mathfrak{c}}$ as an integral over the cycle $H(\mathfrak{c})$ defined in Theorem 4.14. This cycle (its homology class) is stable by the transformation $e^{u_{i}}=1+z_{i}$ which is close to the identity. Thus define the following function on $U$ :

$$
\begin{aligned}
\mathcal{F}_{B}(g, h)(z)= & \frac{\prod_{i=1}^{n}\left(1+z_{i}\right)^{h_{i}+2 n-i-1} \times \prod_{i=1}^{n} g_{i}^{h_{i}}}{\prod_{i=1}^{n}\left(1+z_{i}-g_{i}\right) \times \prod_{1 \leq i<j \leq n}\left(1+z_{i}-g_{i} g_{j}\left(1+z_{j}\right)\right)} \\
& \times \frac{1}{\prod_{1 \leq i<j \leq n}\left(1+z_{i}\right)\left(1+z_{j}\right)-g_{i} g_{j}} .
\end{aligned}
$$

Performing the change of variables $e^{u_{i}}=1+z_{i}$ on the function $F_{B}(g, h)(u)$ and computing the Jacobian, Theorem 3.3 becomes: 
Theorem 8.5. Let $\mathfrak{c}$ be a chamber of $\mathcal{C}\left(\mathcal{B}_{n}\right)$.

- For any $v \in \overline{\mathfrak{c}}$, we have

$$
\operatorname{vol}_{\mathbb{Z}, \mathcal{B}_{n}}(v)=\mathrm{JK}_{\mathfrak{c}}\left(J_{B}(v)\right) .
$$

- For any $h \in V_{\mathbb{Z}} \cap \overline{\mathfrak{c}}$, the value of the partition function is given by

$$
N_{\mathcal{B}_{n}}(h)=\sum_{g \in \Gamma} \frac{1}{(2 \pi \sqrt{-1})^{n}} \int_{H(\mathfrak{c})} \mathcal{F}_{B}(g, h)(z) d z .
$$

As in the case of type $A$, in order to implement these formulas we first have to describe the set $\mathcal{P}\left(v, \mathcal{B}_{n}\right)$ (Section 8.2), then we explain how the integral over the cycle $H(\mathfrak{c})$ is calculated similarly to an iterated residue formula associated to these paths (Section 8.3), using an estimate of the order of poles. Finally we explain how these computations fit together to get a global procedure for the Kostant partition function for $B_{n}$ (Section 8.4).

\subsection{The Search for Maximal Proper Nested Sets}

A height function is

$$
\operatorname{ht}(v)=\sum_{i=1}^{n}(n+1-i) v_{i}
$$

which takes value 1 on all simple roots. We deform ht later in order to have a function taking different values on roots.

We now proceed to describe hyperplanes for $\mathcal{B}_{n}$. If $P=\left[P^{+}, P^{-}\right]$are two disjoints subsets of $\{1,2, \ldots, n\}$, we denote by $\left\langle u_{P}, v\right\rangle$ the linear form $\sum_{i \in P^{+}} v_{i}-\sum_{j \in P^{-}} v_{j}$. Consider the hyperplane

$$
H_{P}=\left\{v \in V,\left\langle u_{P}, v\right\rangle=0\right\}
$$

in $V$. It is equal to the hyperplane determined by the reverse list $\left[P^{-}, P^{+}\right]$. Thus to each set $P=\left\{P^{+}, P^{-}\right\}$of two disjoint sets $P^{+}, P^{-}$such that at least one is non-empty, we associate a hyperplane $H_{P}$.

We denote by $Z$ the complement of $P^{+} \cup P^{-}$in $\{1,2, \ldots, n\}$ and by $\mathcal{B}(Z)$ the subset of $\mathcal{B}_{n}$ defined by

$$
\mathcal{B}(Z)=\left\{e_{i} \mid i \in Z\right\} \cup\left\{e_{i} \pm e_{j} \mid 1 \leq i<j \leq n ; i, j \in Z\right\} .
$$

This is the positive root system $B_{|Z|}$, with the positivity induced by the lexicographic order.

Let $\mathcal{K}\left(P^{+}, P^{-}\right)$be the subset of $\mathcal{B}_{n}$ defined by

$$
\begin{aligned}
\left\{e_{i}-e_{j} \mid 1 \leq i<j \leq n ; i, j \in P^{+}\right\} & \cup\left\{e_{i}+e_{k} \mid i \in P^{+}, k \in P^{-}\right\} \\
& \cup\left\{e_{k}-e_{\ell} \mid 1 \leq k<\ell \leq n ; k, \ell \in P^{-}\right\} .
\end{aligned}
$$


Note that by defining $f_{i}=e_{i}$ if $i \in P^{+}$and $f_{k}=-e_{\mid P^{-\mid-k+1}}$ if $k \in P^{-}$, the set $\mathcal{K}\left(P^{+}, P^{-}\right)$coincides with

$$
\begin{aligned}
\left\{f_{i}-f_{j} \mid 1 \leq i<j \leq n ; i, j \in P^{+}\right\} & \cup\left\{f_{i}-f_{k} \mid i \in P^{+}, k \in P^{-}\right\} \\
& \cup\left\{f_{k}-f_{\ell} \mid 1 \leq k<\ell \leq n ; k, \ell \in P^{-}\right\} .
\end{aligned}
$$

Thus the set $\mathcal{K}\left(P^{+}, P^{-}\right)$is a positive root system of type $A_{\left|P^{+}\right|+\left|P^{-}\right|-1}$. However, the positivity is induced by the lexicographic order on $P^{+}$and the reverse lexicographic order on $P^{-}$. Observe also that $H_{P}$ is the vector space spanned by $\mathcal{K}\left(P^{+}, P^{-}\right) \cup \mathcal{B}(Z)$.

\section{Lemma 8.6.}

- The hyperplane $H_{P}$ is a $\mathcal{B}_{n}$-admissible hyperplane and every $\mathcal{B}_{n}$-admissible hyperplane is of this form.

- The set $\mathcal{B}_{n} \cap H_{P}$ is the union of $\mathcal{B}(Z)$ and $\mathcal{K}\left(P^{+}, P^{-}\right)$.

Proof. Let $H$ be a $\mathcal{B}_{n}$-admissible hyperplane. We prove that $H$ is of the form $H_{P}$ by induction on $n$, the case $n=2$ being trivial. Let $\alpha$ be a root in $H$. There are three possibilities for $\alpha$ : up to renumbering roots, we can consider the cases $\alpha=e_{n}$, $\alpha=e_{n-1}-e_{n}$ and $\alpha=e_{n-1}+e_{n}$.

In the first case, the map $q$ sending $e_{i}$ to $e_{i}$ if $i<n$ and $e_{n}$ to 0 maps the set $\mathcal{B}_{n} \backslash\{\alpha\}$ to $\mathcal{B}_{n-1}$. The space $H / \mathbb{R} \alpha$ becomes a $\mathcal{B}_{n-1}$-admissible hyperplane. It is thus determined by $P^{\prime}=\left[P^{\prime+}, P^{\prime-}\right]$, where $P^{\prime+}$ and $P^{\prime-}$ are two disjoint sets contained in $\{1,2, \ldots, n-1\}$. Then the hyperplane $H$ is equal to the hyperplane determined by $\left[P^{\prime+}, P^{\prime-}\right]$.

In the second case, the map $q$ sending $e_{i}$ to $e_{i}$ if $i<n$ and $e_{n}$ to $e_{n-1}$ sends the set $\mathcal{B}_{n} \backslash\{\alpha\}$ to $\mathcal{B}_{n-1}$. The space $H / \mathbb{R} \alpha$ becomes a $\mathcal{B}_{n-1}$-admissible hyperplane. It is thus determined by $P^{\prime}=\left[P^{\prime+}, P^{\prime-}\right.$ ]. If neither $P^{\prime+}$ nor $P^{\prime-}$ contain $n-1$, the hyperplane $H$ is equal to the hyperplane determined by $\left[P^{\prime+}, P^{\prime-}\right]$. Otherwise assume that, for example, $P^{\prime}+$ contains $n-1$. Then the hyperplane $H$ is equal to the hyperplane determined by $\left[P^{+}, P^{-}\right]$, where $P^{+}=P^{\prime} \cup\{n\}$ and $P^{-}=P^{\prime-}$.

The third case is treated similarly.

We now give a description of our algorithm computing MNSs. We describe a vector as an array $v=\left[v_{1}, v_{2}, \ldots, v_{n}\right]$. To check if $v$ is in the cone $\mathcal{C}\left(\mathcal{B}_{n}\right)$, we need to verify if $\sum_{j=1}^{i} v_{j} \geq 0$ for $1 \leq i \leq n$. This is done by the procedure CheckBvector, which returns the answer true or false.

For the system $\mathcal{B}_{n}$ the highest $\operatorname{root} \theta^{B}(n)$ is equal to

$$
\theta^{B}(n)=[1,1,0,0,0, \ldots, 0] .
$$

We recall here that $P$ is divided in two sets $P^{+} \cup P^{-}$, one of them being non-empty. The first task is to list the hyperplanes in $\operatorname{Hyp}\left(v, \mathcal{B}_{n}\right)$. This set of hyperplanes is obtained by the command line AllPossibleBwalls $(\mathrm{v})$. The input of this procedure is the vector $v$. The output is a set of elements $P=\left\{P^{+}, P^{-}\right\}$, where $P^{+}=\left[i_{1}, i_{2}, \ldots, i_{p}\right]$ and $P^{-}=$ $\left[j_{1}, j_{2}, \ldots, j_{q}\right]$ are two ordered disjoint lists made from indices taken in $\{1, \ldots, n\}$, with at least one of $P^{+}$or $P^{-}$being non-empty. Let $\left\langle u_{P}, v\right\rangle=\sum_{i \in P^{+}} v_{i}-\sum_{j \in P^{-}} v_{j}$ be the 
normal vector to $H_{P}$. Then as stated in Lemma 5.2 we need to test if $\left\langle u_{P}, \theta^{B}(n)\right\rangle$ is not zero and if $\left\langle u_{P}, v\right\rangle \times\left\langle u_{P}, \theta^{B}(n)\right\rangle$ is non-negative.

We then construct the vector

$$
\operatorname{proj}_{H}(v)=v-\frac{\left\langle u_{P}, v\right\rangle}{\left\langle u_{P}, \theta^{B}(n)\right\rangle} \theta^{B}(n) .
$$

This vector is represented as $\left\{\left[v_{1}, P^{+}\right],\left[v_{2}, P^{-}\right],[w, Z]\right\}$. The sum of coordinates of $v_{1}$ is equal to the sum of the coordinates of $v_{2}$. Now $Z$ is the ordered list $\left[k_{1}, k_{2}, \ldots, k_{\ell}\right]$ of complementary indices to $P^{+} \cup P^{-}$and

$$
w=\left[\operatorname{proj}_{H}(v)\left[k_{1}\right], \ldots, \operatorname{proj}_{H}(v)\left[k_{\ell}\right]\right] .
$$

Note that the equations of the cone $\mathcal{C}\left(\mathcal{K}\left(P^{+}, P^{-}\right)\right)$can be given in the convenient form $v_{1} \oplus v_{2} \in \mathcal{C}\left(\mathcal{K}\left(P^{+}, P^{-}\right)\right)$if and only if CheckBvector $\left(\mathrm{v}_{1}\right)$ and CheckBvector $\left(\mathrm{v}_{2}\right)$ are true. Equations of the cone $\mathcal{C}(\mathcal{B}(Z))$ are given in the form $w \in \mathcal{C}(\mathcal{B}(Z))$ if and only if CheckBvector(w) is true.

Thus the condition that $H$ is in $\operatorname{Hyp}\left(v, \mathcal{B}_{n}\right)$ is equivalent to the series of conditions:

$$
\begin{aligned}
\left\langle u_{P}, \theta^{B}(n)\right\rangle & \neq 0, \\
\left\langle u_{P}, v\right\rangle \times\left\langle u_{P}, \theta^{B}(n)\right\rangle & \geq 0, \\
\text { CheckBvector }\left(\mathrm{v}_{1}\right) & =\text { true, } \\
\text { CheckBvector }\left(\mathrm{v}_{2}\right) & =\text { true, } \\
\text { CheckBvector }(\mathrm{w}) & =\text { true. }
\end{aligned}
$$

These five conditions are checked by the command line CheckBwall (v, H), that gives an answer true or false.

This achieves the description of the procedure AllPossibleBwalls. We now have to perform the next step of our algorithm. As for type $A$ we build MNSs iteratively. At each step we get a set of partial MNSs, to which we recursively apply our algorithm. Note that after Lemma 8.6 the intersection of a $\mathcal{B}_{n}$-admissible hyperplane $H_{P}$ with $\mathcal{B}_{n}$ is the union of a system of type $A$ and a system of type $B$.

The part of the MNS coming from the subsystem of type $A$ is computed with the procedure AddAnests. It performs a reordering of the result of a call to the procedure MNSS described in Section 7.2.

The part of the MNS coming from the subsystem of type $B$ is computed with the procedure Bsplits, calling the previously described procedure AllPossibleBwalls.

\subsection{Residues Associated to Maximal Proper Nested Sets}

An MPNS $M$ gives rise to an ordered basis $\alpha_{i}$, and a cycle $H(M)$. We need to compute

$$
\int_{H(M)} \mathcal{F}_{B}(g, h)(z) d z
$$


where

$$
H(M):=\left\{z,\left|\left\langle\alpha_{i}, z\right\rangle\right|=\varepsilon_{i}\right\} .
$$

The function $z \mapsto \mathcal{F}_{B}(g, h)(z)$ is deduced from the function $F_{B}(g, h)(u)$ in the space $\widehat{R}_{\mathcal{A}}$ by the change of variable $e^{u_{i}}=1+z_{i}$. Thus its denominator is a product of factors, either of the form $z_{i}$ corresponding to the root $u_{i}$, or of the form $z_{i}-z_{j}$ corresponding to the root $u_{i}-u_{j}$ or $z_{i}+z_{j}+z_{i} z_{j}$ corresponding to the root $u_{i}+u_{j}$. We denote by $u(z)$ the point with coordinates $u_{i}$ satisfying $e^{u_{i}}=1+z_{i}$.

We start integrating our function $\mathcal{F}_{B}(g, h)(z)$ over the smaller circle $\left|\left\langle\alpha_{1}, z\right\rangle\right|=\varepsilon_{1}$ keeping the other variables fixed. By our condition on the cycle, the function we integrate has poles on the domain $\left|\left\langle\alpha_{1}, z\right\rangle\right| \leq \varepsilon_{1}$ only when $\alpha_{1}(u(z))=0$. If $\alpha_{1}(u(z))=u_{i}-u_{j}$ or $\alpha_{1}(u(z))=u_{i}$, the poles are obtained for $z_{i}=z_{j}$ or $z_{i}=0$. If $\alpha_{1}(u(z))=u_{i}+u_{j}$, the pole on the domain $\left|\left\langle\alpha_{1}, z\right\rangle\right| \leq \varepsilon_{1}$ is obtained for $z_{i}=-z_{j} /\left(1+z_{j}\right)$. Thus we compute the integral over the circle by the residue theorem in one variable, and proceed. From the general theory, the poles of the function we obtain, replacing $z_{i}$ by one of the values above, are again of the same form with respect to the remaining variables, as is easily checked.

As in case $A_{n-1}$, for a root $\alpha=u_{i}$ (resp. $\alpha=u_{i} \pm u_{j}$ ) we can replace after taking the residue at $\alpha=0$ the variable $z_{i}$ by 0 (resp. by $\mp z_{j}$ ) in all other roots. Thus we eliminate the variable $z_{i}$. The procedure FormalPathB produces the ordered path resulting from all these substitutions.

In the case of type $B$ we compute the residue by directly checking the order of the pole at $\alpha=0$, and then using differentiation. The program works in the same way with parameters. The function obtained is locally polynomial with polynomial coefficients depending of the parity of the integers $h_{i}$.

\subsection{The Procedure MNS_KostantB}

We finish the section dedicated to $B_{n}$ by giving the global outline of the procedure MNS_Kostant $\mathrm{B}(\mathrm{v})$ computing the Kostant partition number of a vector $v$ lying in the root lattice of $\mathcal{B}_{n}$. We begin by slightly deforming $v$ so that it lies on no wall, by setting $\mathrm{v}^{\prime}:=\operatorname{DefVectorB}(\mathrm{v}, \mathrm{n})$. We then compute all MNSs for $v^{\prime}$ with the call B_MNSs $\left(\mathrm{v}^{\prime}\right)$ (Section 8.2). For every MNS $M$, we extract the list $R$ of highest roots of its irreducible components by setting $\mathrm{R}:=$ BthetaMnS(M). We sort these roots by their height with the command line $\mathrm{R}^{\prime}:=$ BorderThetas $(\mathrm{R}, \mathrm{n})$. We then transform the list of roots $R^{\prime}$ into a path $p$ by setting $\mathrm{p}:=$ FormalPathB $\left(\mathrm{R}^{\prime}\right)$.

Now note that our procedures are designed to take residues along positive roots, using the fact that res ${ }_{-\alpha}=-\operatorname{res}_{\alpha}$ for any root $\alpha$. The sign that appears (more precisely -1 to the power the number of negative roots in the path $p$ ) is computed with the procedure PathSign(p, n).

Then for every $g$ in $\Gamma$ we do the following. The iterated residue along the path $p$ and for $g$ is obtained by the command line OneIteratedBresidue(p, g, v, n). We briefly describe its implementation. We first compute the Kostant fraction (second item of Definition 7.1, procedure KostantFunctionB). Then for every root of the path we apply the procedure ComputeoneResidue (Section 8.3) and update the order of the pole with a procedure named OrderPoleB. 
Finally, summing all products PathSign $(\mathrm{p}, \mathrm{n}) \times$ OneIteratedBresidue $(\mathrm{p}, \mathrm{g}, \mathrm{v}, \mathrm{n})$ over the sets of $g$ 's and of $M$ 's, we get the desired result.

Remark 8.7. Let us fix a list $R^{\prime}=\left[\alpha_{1}, \ldots, \alpha_{n}\right]$ of ordered roots coming from an MNS, and an element $g$. We say that $R^{\prime}$ and $g$ are compatible if the following condition is satisfied. If indices of monomial(s) of $\alpha_{k}$ have not yet occurred among indices of roots $\alpha_{\ell}$ with $\ell<k$, then $g$ must satisfy $g^{\alpha_{k}}=1$ (that is $g_{i} g_{j}^{ \pm 1}=1$ if $\alpha_{k}=e_{i} \pm e_{j}$ and $g_{i}=1$ if $\alpha_{k}=e_{i}$ ). Note that the iterated residue for $g$ and for the path $p$ associated to $R^{\prime}$ is zero if $g$ and $R^{\prime}$ are not compatible. Hence summing only over $g$ 's that are compatible with a given list $R^{\prime}$ saves useless computations. The check of compatibility is performed by the procedure ListAndGAreCompatible $\left(R^{\prime}, g, n\right)$.

\section{Type $C_{n}$}

Consider a vector space $V$ with basis $e_{1}, e_{2}, \ldots, e_{n}$. We choose on $V$ the standard Lebesgue measure $d h$. Let

$$
\mathcal{C}_{n}=\left\{2 e_{i} \mid 1 \leq i \leq n\right\} \cup\left\{e_{i}-e_{j} \mid 1 \leq i<j \leq n\right\} \cup\left\{e_{i}+e_{j} \mid 1 \leq i<j \leq n\right\} .
$$

Then $\mathcal{C}_{n}$ is a positive roots system of type $C_{n}$, and generates $V$. The number of elements in $\mathcal{C}_{n}$ is $N=n^{2}$. Note that elements of $\mathcal{C}_{n}$ and $\mathcal{B}_{n}$ are proportional, so they determine the same hyperplane arrangement and the same chambers.

Let $L$ be the lattice defined by $\mathbb{Z} e_{1} \oplus \mathbb{Z} e_{2} \oplus \cdots \oplus \mathbb{Z} e_{n}$. We remark that the lattice $V_{\mathbb{Z}}$ generated by $\mathcal{C}_{n}$ is the sublattice of index 2 in $L$ consisting of all elements $v=$ $\left[v_{1}, v_{2}, \ldots, v_{n}\right]$ with integral coordinates and such that the sum $\sum_{i=1}^{n} v_{i}$ is an even integer. A $\mathbb{Z}$-basis of $V_{\mathbb{Z}}$ is, for example,

$$
\mathbb{Z}\left(e_{1}-e_{n}\right) \oplus \mathbb{Z}\left(e_{2}-e_{n}\right) \oplus \cdots \oplus \mathbb{Z}\left(e_{n-1}-e_{n}\right) \oplus \mathbb{Z}\left(2 e_{n}\right),
$$

$\operatorname{sov} \operatorname{vol}\left(V / V_{\mathbb{Z}}\right)=2$.

The dual lattice $U_{\mathbb{Z}}$ is the lattice of vectors $\gamma=\left(\gamma_{1}, \gamma_{2}, \ldots, \gamma_{n}\right)$ such that $\gamma_{i}$ are half integers and such that $\gamma_{i}+\gamma_{j}$ is an integer for all $i, j$. The set $U_{\mathbb{Z}} / \mathbb{Z} e_{1} \oplus \cdots \oplus \mathbb{Z} e_{n}$ is of cardinality 2 with representative elements $(0,0, \ldots, 0,0)$ and $\left(\frac{1}{2}, \ldots, \frac{1}{2}\right)$.

As before, we identify the torus $\tilde{T}=U /\left(\mathbb{Z} e_{1} \oplus \cdots \oplus \mathbb{Z} e_{n}\right)=\mathbb{R}^{n} / \mathbb{Z}^{n}$ with $\left(S^{1}\right)^{n}$ by

$$
\left(u_{1}, u_{2}, \ldots, u_{n}\right) \mapsto\left(e^{2 \pi \sqrt{-1} u_{1}}, \ldots, e^{2 \pi \sqrt{-1} u_{n}}\right) .
$$

Then

$$
T=\tilde{T} /\{ \pm 1\}=U / U_{\mathbb{Z}}
$$

Let $G$ be a representative of $g=\left(g_{1}, g_{2}, \ldots, g_{n}\right) \in \tilde{T}$ and $h=\sum_{i=1}^{n} h_{i} e_{i}$ in $V_{\mathbb{Z}}$. Then $e^{\langle h, 2 \pi \sqrt{-1} G\rangle}$ is equal to $\prod_{i=1}^{n} g_{i}^{h_{i}}=g^{h}$. This function is well defined on $T=\tilde{T} /\{ \pm 1\}$ since $\sum_{i=1}^{n} h_{i}$ is even.

For $\sigma$ a basic subset of $\mathcal{C}_{n}$, define

$$
\tilde{T}(\sigma)=\left\{g \in \tilde{T} \mid e^{\langle\alpha, 2 \pi \sqrt{-1} G\rangle}=1 \text { for all } \alpha \in \sigma\right\} .
$$

As the set $\mathcal{C}_{n}$ is not unimodular, sets $\tilde{T}(\sigma)$ are not reduced to 1 . By induction on $n$, we prove the following lemma. 
Lemma 9.1. Let $\sigma$ be a basic subset of $\mathcal{C}_{n}$. Then $\tilde{T}(\sigma) \subset\{ \pm 1\}^{n}$.

Let $v=\sum_{i=1}^{n} v_{i} e_{i} \in V$ be a vector with real coordinates and let $h=\sum_{i=1}^{n} h_{i} e_{i} \in$ $V$ be a vector with integral coordinates and such that $\sum_{i=1}^{n} h_{i}$ is even. We compute the normalized volume of $\Pi_{\mathcal{C}_{n}}(v)$ and the number of integral points in $\Pi_{\mathcal{C}_{n}}(h)$ using Theorem 3.3. We use the JK residue with respect to the measure $d h$ associated to the basis $e_{1}, e_{2}, \ldots, e_{n}$. However, the normalized volume $\operatorname{vol}_{\mathbb{Z}, \mathcal{C}_{n}}(h)$ is computed for the measure determined by the lattice spanned by $\mathcal{C}_{n}$ which is of index 2 in $\bigoplus_{i=1}^{n} \mathbb{Z} e_{i}$.

We introduce the function $J_{C}(v)$ on $U$ defined by

$$
J_{C}(v)(u)=\frac{e^{\sum_{i=1}^{n} u_{i} v_{i}}}{\prod_{i=1}^{n} 2 u_{i} \prod_{1 \leq i<j \leq n}\left(u_{i}-u_{j}\right) \prod_{1 \leq i<j \leq n}\left(u_{i}+u_{j}\right)} .
$$

For $g=\left(g_{1}, g_{2}, \ldots, g_{n}\right) \in\{ \pm 1\}^{n}$ the Kostant fraction (3) is the function on $U$ defined by

$$
\begin{aligned}
F_{C}(g, h)(u)= & \frac{\prod_{i=1}^{n} g_{i}^{h_{i}} e^{\sum_{i=1}^{n} u_{i} h_{i}}}{\prod_{i=1}^{n}\left(1-e^{-2 u_{i}}\right) \times \prod_{1 \leq i<j \leq n}\left(1-g_{i}^{-1} g_{j} e^{-\left(u_{i}-u_{j}\right)}\right)} \\
& \times \frac{1}{\prod_{1 \leq i<j \leq n}\left(1-g_{i}^{-1} g_{j}^{-1} e^{-\left(u_{i}+u_{j}\right)}\right)} .
\end{aligned}
$$

Theorem 9.2. Let $\mathfrak{c}$ be a chamber of $\mathcal{C}\left(\mathcal{C}_{n}\right)$.

- For any $v \in \overline{\mathfrak{c}}$, we have

$$
\operatorname{vol}_{\mathbb{Z}, \mathcal{C}_{n}}(v)=2 \mathrm{JK}_{\mathfrak{c}}\left(J_{C}(v)\right) .
$$

- For any vector $h \in V_{\mathbb{Z}} \cap \overline{\mathfrak{c}}$ with integral coordinates such that $\sum_{i=1}^{n} h_{i}$ is even, the value of the partition function is given by

$$
N_{\mathcal{C}_{n}}(h)=\sum_{g \in\{ \pm 1\}^{n}} \mathrm{JK}_{\mathfrak{c}}\left(F_{C}(g, h)\right) .
$$

In the second formula, there should be a multiplication by a factor 2 as the volume of the fundamental domain of the lattice spanned by $\mathcal{C}_{n}$ is 2 . However, we should sum only on $T=\tilde{T} /\{ \pm 1\}$. Thus the two factors of 2 compensate each other. In fact, we indeed sum over $T$ represented as $\{ \pm 1\}^{n-1} \times\{1\}$ and multiply the result by the constant 2 .

As in the case of $B_{n}$, we use the change of variable $1+z_{i}=e^{u_{i}}$ to compute more easily the formula for $N_{\mathcal{C}_{n}}(h)$. As explained in the case of $B_{n}$ we need to use the integral formulation of the JK residue. Thus define

$$
\begin{aligned}
\mathcal{F}_{C}(g, h)(z)= & \frac{\prod_{i=1}^{n}\left(1+z_{i}\right)^{h_{i}+2 n-i} \times \prod_{i=1}^{n} g_{i}^{h_{i}}}{\prod_{i=1}^{n}\left(\left(1+z_{i}\right)^{2}-1\right) \times \prod_{1 \leq i<j \leq n}\left(1+z_{i}-g_{i} g_{j}\left(1+z_{j}\right)\right)} \\
& \times \frac{1}{\prod_{1 \leq i<j \leq n}\left(1+z_{i}\right)\left(1+z_{j}\right)-g_{i} g_{j}} .
\end{aligned}
$$

Performing the change of variables $e^{u_{i}}=1+z_{i}$ on the function $F_{C}(g, h)(u)$ and computing the Jacobian, Theorem 3.3 becomes: 
Theorem 9.3. Let $\mathfrak{c}$ be a chamber of $\mathcal{C}\left(\mathcal{C}_{n}\right)$.

- For any $v \in \overline{\mathfrak{c}}$, we have

$$
\operatorname{vol}_{\mathbb{Z}, \mathcal{C}_{n}}(v)=2 \operatorname{JK}_{\mathfrak{c}}\left(J_{C}(v)\right) .
$$

- For any vector $h \in V_{\mathbb{Z}} \cap \overline{\mathfrak{c}}$ with integral coordinates $h_{i}$ with $\sum_{i=1}^{n} h_{i}$ even, the value of the partition function is given by

$$
N_{\mathcal{C}_{n}}(h)=\sum_{g \in\{ \pm 1\}^{n}} \frac{1}{(2 \pi \sqrt{-1})^{n}} \int_{H(\mathfrak{c})} \mathcal{F}_{C}(g, h)(z) d z .
$$

Similarly we sum over $T$ represented as $\{ \pm 1\}^{n-1} \times\{1\}$ and multiply the result by the constant 2.

The cycle $H(\mathfrak{c})$ associated to a chamber $\mathfrak{c}$ containing a regular element $v=\sum_{i=1}^{n} v_{i} e_{i}$ is the same cycle that we computed in the preceding section for $B_{n}$. Hence we can reuse most of procedures from type $B_{n}$. Paths are the same, and the residue calculations are the same. More precisely, the only two changes are in the computation of the set $G$ (procedure $\mathrm{GC}(\mathrm{n}))$ and in the computation of the Kostant function (procedure UCKostant). This terminates the case of $C_{n}$.

\section{Type $D_{n}$}

\subsection{The Formulas to Be Implemented}

Consider a vector space $V$ with basis $e_{1}, e_{2}, \ldots, e_{n}$. We choose the standard Lebesgue measure $d h$. Let

$$
\mathcal{D}_{n}=\left\{e_{i}-e_{j} \mid 1 \leq i<j \leq n\right\} \cup\left\{e_{i}+e_{j} \mid 1 \leq i<j \leq n\right\} .
$$

Then $\mathcal{D}_{n}$ is a positive roots system of type $D_{n}$, and generates $V$. The number of elements in $\mathcal{D}_{n}$ is $N=n^{2}-n$.

We remark that the lattice $V_{\mathbb{Z}}$ generated by roots of $\mathcal{D}_{n}$ is the same lattice as the one generated by the roots of $\mathcal{C}_{n}$. It is of index 2 in $L:=\mathbb{Z} e_{1} \oplus \mathbb{Z} e_{2} \oplus \cdots \oplus \mathbb{Z} e_{n}$ and consists of elements $v=\left[v_{1}, v_{2}, \ldots, v_{n}\right]$ with integral coordinates such that the sum $\sum_{i=1}^{n} v_{i}$ is an even integer. The group $T=U / U_{\mathbb{Z}}$ is thus the quotient of $\tilde{T}=U / \mathbb{Z} e_{1} \oplus \cdots \oplus \mathbb{Z} e_{n}$, obtained by identifying $g$ and $-g$, that is $T=\tilde{T} /\{ \pm 1\}$. As in Section 9 , we identify the torus $\tilde{T}=U /\left(\mathbb{Z} e_{1} \oplus \cdots \oplus \mathbb{Z} e_{n}\right)=\mathbb{R}^{n} / \mathbb{Z}^{n}$ to $\left(S^{1}\right)^{n}$ by

$$
\left(u_{1}, u_{2}, \ldots, u_{n}\right) \mapsto\left(e^{2 \pi \sqrt{-1} u_{1}}, \ldots, e^{2 \pi \sqrt{-1} u_{n}}\right) .
$$

Consider the set $\Gamma=\{ \pm 1\}^{n} \subset\left(S^{1}\right)^{n}$. For $\sigma$ a basic subset of $\mathcal{D}_{n}$, define

$$
\tilde{T}(\sigma)=\left\{g \in \tilde{T} \mid e^{\langle\alpha, 2 \pi \sqrt{-1} G\rangle}=1 \text { for all } \alpha \in \sigma\right\} .
$$

Lemma 10.1. Let $\sigma$ be a basic subset of $\mathcal{D}_{n}$. Then $\tilde{T}(\sigma)$ is contained in $\Gamma$. 
Proof. Basic subsets of $\mathcal{D}_{n}$ are basic subsets of $\mathcal{C}_{n}$ so that we can choose the same set $\Gamma=\{ \pm 1\}^{n}$.

Let $v=\sum_{i=1}^{n} v_{i} e_{i} \in V$ be a vector with real coordinates and let $h=\sum_{i=1}^{n} h_{i} e_{i} \in$ $V$ be a vector with integral coordinates and such that $\sum_{i=1}^{n} h_{i}$ is even. We compute the normalized volume of $\Pi_{\mathcal{D}_{n}}(v)$ and the number of integral points in $\Pi_{\mathcal{D}_{n}}(h)$ using Theorem 3.3.

Thus we introduce the function $J_{D}(v)$ on $U$ defined by

$$
J_{D}(v)(u)=\frac{e^{\sum_{i=1}^{n} u_{i} v_{i}}}{\prod_{1 \leq i<j \leq n}\left(u_{i}-u_{j}\right) \prod_{1 \leq i<j \leq n}\left(u_{i}+u_{j}\right)} .
$$

For $g=\left(g_{1}, g_{2}, \ldots, g_{n}\right) \in\{ \pm 1\}^{n}$ the Kostant fraction (3) is the function on $U$ defined by

$$
\begin{aligned}
F_{D}(g, h)(u)= & \frac{\prod_{i=1}^{n} g_{i}^{h_{i}} \times e^{\sum_{i=1}^{n} u_{i} h_{i}}}{\prod_{1 \leq i<j \leq n}\left(1-g_{i}^{-1} g_{j} e^{-\left(u_{i}-u_{j}\right)}\right)} \\
& \times \frac{1}{\prod_{1 \leq i<j \leq n}\left(1-g_{i}^{-1} g_{j}^{-1} e^{-\left(u_{i}+u_{j}\right)}\right)} .
\end{aligned}
$$

We then have

Theorem 10.2. Let $\mathfrak{c}$ be a chamber of $\mathcal{C}\left(\mathcal{D}_{n}\right)$.

- For any $v \in \overline{\mathfrak{c}}$, we have

$$
\operatorname{vol}_{\mathbb{Z}, \mathcal{D}_{n}}(v)=2 \operatorname{JK}_{\mathfrak{c}}\left(J_{D}(v)\right) .
$$

- For any vector $h \in V_{\mathbb{Z}} \cap \overline{\mathfrak{c}}$ with integral coordinates such that $\sum_{i=1}^{n} h_{i}$ is even, the value of the partition function is given by

$$
N_{\mathcal{D}_{n}}(h)=\sum_{g \in\{ \pm 1\}^{n}} \mathrm{JK}_{\mathfrak{c}}\left(F_{D}(g, h)\right) .
$$

We use the change of variable $1+z_{i}=e^{u_{i}}$ to compute more easily the formula for $N_{\mathcal{D}_{n}}(h)$ and thus introduce integration over a cycle. Thus define

$$
\begin{aligned}
\mathcal{F}_{D}(g, h)(z)= & \frac{\prod_{i=1}^{n}\left(1+z_{i}\right)^{h_{i}+2 n-i-2} \times \prod_{i=1}^{n} g_{i}^{h_{i}}}{\prod_{1 \leq i<j \leq n}\left(1+z_{i}-g_{i} g_{j}\left(1+z_{j}\right)\right)} \\
& \times \frac{1}{\prod_{1 \leq i<j \leq n}\left(1+z_{i}\right)\left(1+z_{j}\right)-g_{i} g_{j}} .
\end{aligned}
$$

After performing the change of variables $e^{u_{i}}=1+z_{i}$ on the function $F_{D}(g, h)(u)$ and after computing the Jacobian, Theorem 3.3 becomes: 
Theorem 10.3. Let $\mathfrak{c}$ be a chamber of $\mathcal{C}\left(\mathcal{D}_{n}\right)$.

- For any $v \in \overline{\mathfrak{c}}$, we have

$$
\operatorname{vol}_{\mathbb{Z}, \mathcal{D}_{n}}(v)=2 \operatorname{JK}_{\mathfrak{c}}\left(J_{D}(v)\right) .
$$

- For any vector $h \in V_{\mathbb{Z}} \cap \overline{\mathfrak{c}}$ with integral coordinates $h_{i}$ such that $\sum_{i=1}^{n} h_{i}$ is even, the value of the partition function is given by

$$
N\left(\mathcal{D}_{n}, h\right)=\sum_{g \in\{ \pm 1\}^{n}} \frac{1}{(2 \pi \sqrt{-1})^{n}} \int_{H(\mathfrak{c})} \mathcal{F}_{D}(g, h)(z) d z .
$$

As for types $A$ and $B$, in order to implement these formulas we first have to describe the set $\mathcal{P}\left(v, \mathcal{D}_{n}\right)$ (Section 10.2). We finish by explaining the implementation of case $D$ in Section 10.3, using the fact that types $B$ and $D$ are similar.

\subsection{The Search for Maximal Proper Nested Sets}

A height function is

$$
\operatorname{ht}(v)=\sum_{i=1}^{n}(n-i) v_{i}
$$

which takes value 1 on all simple roots. We deform it later in order to have a function taking different values on roots.

We now proceed to describe hyperplanes for $D_{n}$. If $P=\left[P^{+}, P^{-}\right]$are two disjoints subsets of $\{1,2, \ldots, n\}$, we denote by $\left\langle u_{P}, v\right\rangle$ the linear form $\sum_{i \in P^{+}} v_{i}-\sum_{j \in P^{-}} v_{j}$. Consider the hyperplane in $V$ defined by

$$
H_{P}=\left\{v \in V,\left\langle u_{P}, v\right\rangle=0\right\}
$$

and note that it is equal to the hyperplane determined by the reverse list $\left[P^{-}, P^{+}\right]$. Thus to each set $P=\left\{P^{+}, P^{-}\right\}$of two disjoint sets $P^{+}, P^{-}$such that at least one is non-empty, is associated a hyperplane $H_{P}$.

We denote by $Z$ the complement of $P^{+} \cup P^{-}$in $\{1,2, \ldots, n\}$ and by $\mathcal{D}(Z)$ the subset of $\mathcal{D}_{n}$ defined by

$$
\mathcal{D}(Z)=\left\{e_{i} \pm e_{j} \mid 1 \leq i<j \leq n ; i, j \in Z\right\} .
$$

This is the positive roots system of type $D_{|Z|}$, with the positivity induced by the lexicographic order.

Let $\mathcal{K}\left(P^{+}, P^{-}\right)$be the subset of $\mathcal{D}_{n}$ defined by

$$
\begin{aligned}
\left\{e_{i}-e_{j} \mid 1 \leq i<j \leq n ; i, j \in P^{+}\right\} & \cup\left\{e_{i}+e_{k} \mid i \in P^{+}, k \in P^{-}\right\} \\
& \cup\left\{e_{k}-e_{\ell} \mid 1 \leq k<\ell \leq n ; k, \ell \in P^{-}\right\} .
\end{aligned}
$$

As we observed in Section 8.2 for $B_{n}$, by defining $f_{i}=e_{i}$ if $i \in P^{+}$and $f_{k}=-e_{|P|-k+1}$ if $k \in P^{-}$, the set $\mathcal{K}\left(P^{+}, P^{-}\right)$is a positive roots system of type $A_{\left|P^{+}\right|+\mid P^{-\mid-1}}$. Here the 
positivity is induced by the lexicographic order on $P^{+}$and the reverse lexicographic order on $P^{-}$.

Observe also that $H_{P}$ is the vector space spanned by $\mathcal{K}\left(P^{+}, P^{-}\right) \cup \mathcal{D}(Z)$. As before, we prove

\section{Lemma 10.4.}

- The hyperplane $H_{P}$ is a $\mathcal{D}_{n}$-admissible hyperplane and every $\mathcal{D}_{n}$-admissible hyperplane is of this form.

- The set $\mathcal{D}_{n} \cap H_{P}$ is the union of $\mathcal{D}(Z)$ and $\mathcal{K}\left(P^{+}, P^{-}\right)$.

\subsection{The Procedure MNS_KostantD}

Most of procedures from type $B_{n}$ are kept unchanged. More precisely, the iterated residue calculation, the estimate of the order of poles, and the global procedures coordinating computations are exactly the same as for type $B_{n}$.

The only serious adaptations to the case of $D_{n}$ appears in the procedure CheckDvector $(n, v)$. In fact now we check that

$$
\begin{aligned}
v_{1}+\cdots+v_{i} \geq 0 & \text { for } \quad 1 \leq i \leq n-1, \\
v_{1}+\cdots+v_{n-1}+v_{n} \geq 0 & \text { and is even, } \\
v_{1}+\cdots+v_{n-1}-v_{n} \geq 0 & \text { and is even. }
\end{aligned}
$$

Other modifications are in procedures that are parents of CheckDvector. For example the procedure CheckDwall works exactly as CheckBwall, but now calls CheckDvector instead of CheckBvector. See Section 8.2.

\section{Performance of the Programs}

In this section we describe several tests of our programs implementing the above MNS algorithms for types $A_{n}, B_{n}, C_{n}$, and $D_{n}$. The algorithm implementation is made with Maple. Our programs are freely available at www.math.polytechnique.fr/ $\sim$ vergne/work/IntegralPoints.html. We compare our results with the ones obtained by two previous algorithms:

- The Sp (for special permutations) algorithm by Baldoni et al. [2], only for $A_{n}$.

- The implementation LattE of Barvinok's algorithm [15], for every classical algebra.

These two methods also helped us test our algorithms on various examples.

Note that for our programs most of the computation time is spent while computing iterated residues. Indeed, MNS computation is fast and efficient. Note also that most of the memory used by our programs serves to store all fractions that occur in the iterated residue process. The number of MNSs has a great influence on computation time, since we sum over all MNSs. In any case it seems that the deeper a vector is in the cone generated by positive roots, the higher the number of MNSs is. This is morally bound to 


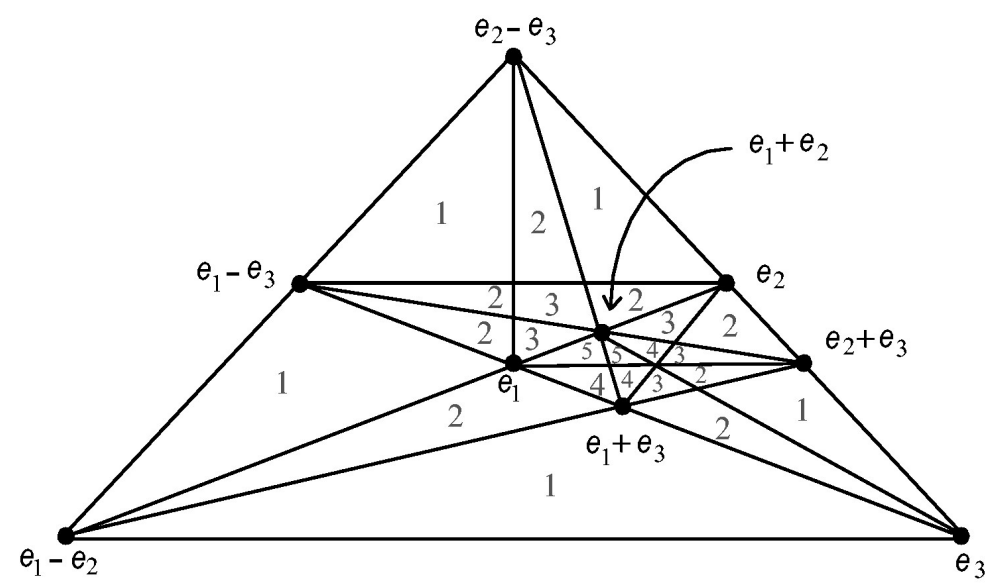

Fig. 9. Number of MNSs containing any vector in a given chamber for $B_{3}$.

the fact that there are more simplicial cones that might contain the vector. In Fig. 9 we attach to every chamber $\mathfrak{c}$ for $B_{3}$ the number of MNSs associated to any vector $v \in \mathfrak{c}$.

Recall that the Sp algorithm relies on sums over a set $\mathrm{Sp}(a)$ of special permutations for a vector $a$. The main advantage of our algorithms is that we compute fewer iterated residues. In fact, the number of MNS seems to be smaller than the number of special permutations that occur, for a given generic example. However, examples at the end of Table 2 show that a number of MNSs considerably smaller than those of Sp's does not lead to a better performance in time computation, even in the extreme case of just one MNS. Indeed, this one residue computation can be very time consuming due to the substitutions $z_{i}=z_{j}$, which takes more time that the substitutions $z_{i}=0$ used in the $\mathrm{Sp}(a)$ algorithm. In the near future we will improve this minor point. The MNSs method should be better and is better in general.

During comparative tests, we figured out that one example in [2] has not been correctly copied from draft. More precisely in their Table 2 for the complete graph $K_{n}$, for the vector

$$
a=(82275,33212,91868,-57457,47254,-64616,94854,-227390)
$$

in the root lattice for $A_{7}$, the correct Kostant partition number is the 93-digit integer

$$
\begin{array}{r}
226040494681135377722281761934040091356424181 \\
242669497614801846058092972975120580334961426497
\end{array}
$$

and not only the first line of 45 digits. The Kostant number and Ehrhart polynomials for this $a$ were computed on a $1 \mathrm{GHz}$ computer in $2.14 \mathrm{~s}$ and $18.54 \mathrm{~s}$, respectively, using 26 special permutations. Now with our programs running on a $1.13 \mathrm{GHz}$ computer these times drop to $1.38 \mathrm{~s}$ and $2.50 \mathrm{~s}$ respectively, using 14 maximal nested sets. Similarly for the biggest example examined in [2], that is for the vector

$$
\begin{aligned}
a= & (46398,36794,92409,-16156,29524,-68385, \\
& 93335,50738,75167,-54015,-285809)
\end{aligned}
$$




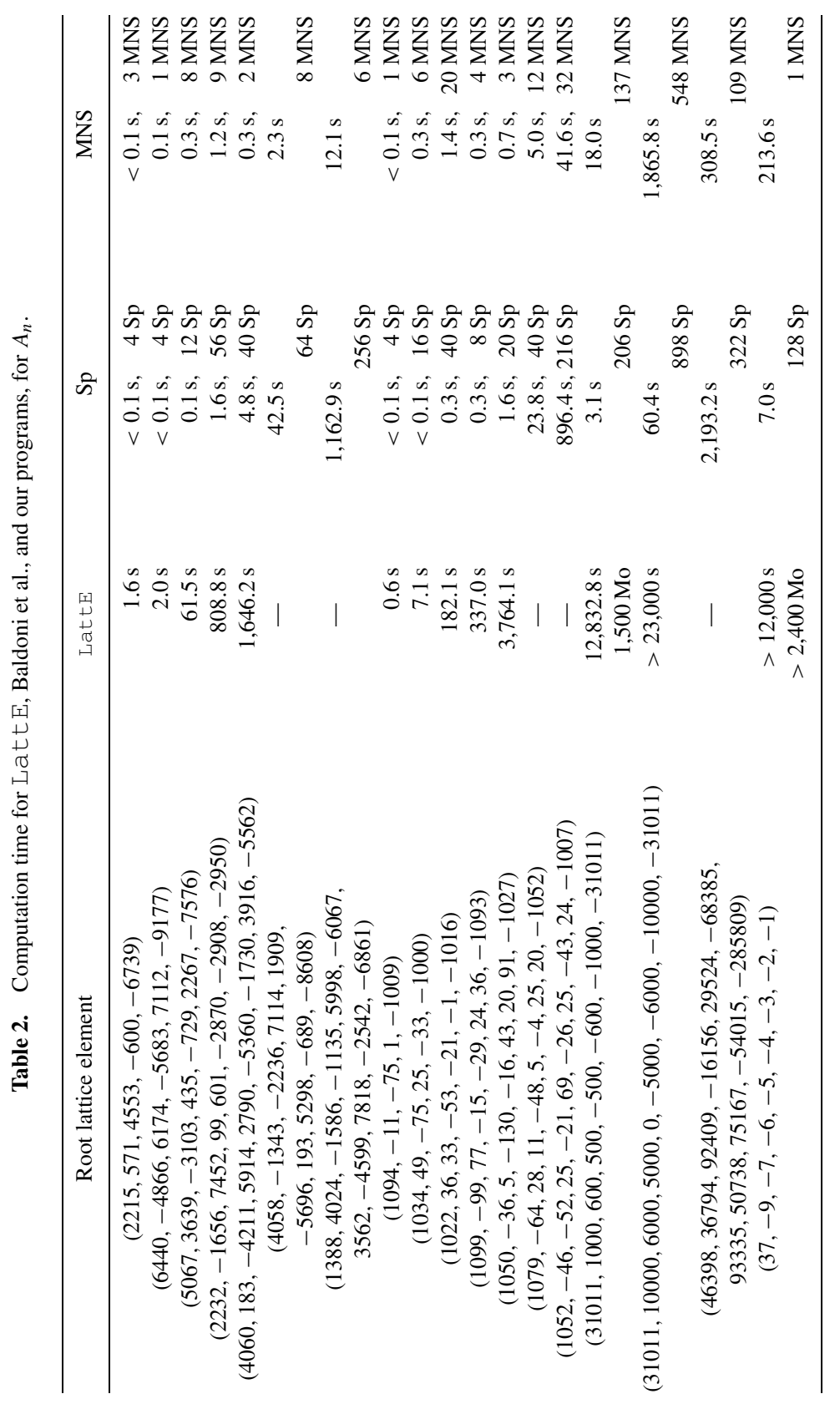


Table 3. Computation time for LattE and our programs, for $B_{n}$.

\begin{tabular}{crr}
\hline Root lattice element & LattE & \multicolumn{1}{c}{ MNS } \\
\hline$(1388,4024,3826)$ & $0.8 \mathrm{~s}$ & $<0.1 \mathrm{~s}$ \\
$(2691,5998,-6067,6184)$ & & $3 \mathrm{MNS}$ \\
$(1585,7818,-2542,-2803,2715)$ & & $0.1 \mathrm{~s}$ \\
$(479,7114,1909,-5696,193,9297)$ & $214.9 \mathrm{~s}$ & $3.0 \mathrm{~s}$ \\
$(1070,1006,-37)$ & & $2 \mathrm{MNS}$ \\
$(1082,947,27,42)$ & & $27.5 \mathrm{~s}$ \\
$(1047,974,20,44,-35)$ & & $8 \mathrm{MNS}$ \\
& & $0.1 \mathrm{~s}$ \\
& & $3 \mathrm{MNS}$ \\
& & $1.2 \mathrm{~s}$ \\
& & $15 \mathrm{MNS}$ \\
& & $21.7 \mathrm{~s}$ \\
& & $51 \mathrm{MNS}$ \\
& & $378.0 \mathrm{~s}$ \\
& & $26 \mathrm{MNS}$ \\
\hline
\end{tabular}

in the root lattice for $A_{10}$, the 189-digit answer was obtained in $2193 \mathrm{~s}$ using 322 special permutations, whereas now we get the same result in $308 \mathrm{~s}$ using 109 MNSs.

Table 2 contains respective performances for $A_{n}$ of LattE, Sp algorithms, and our programs, apart from the last four examples that compare only the last program with ours. Tables 3-5 contain respective performances for $B_{n}, C_{n}$, and $D_{n}$ of LattE and our programs. We also indicated the number of special permutations (Sp) and maximal nested sets (MNS).

Table 4. Computation time for Latt $\mathrm{E}$ and our programs, for $C_{n}$.

\begin{tabular}{crr}
\hline Root lattice element & \multicolumn{1}{c}{ LattE } & \multicolumn{1}{c}{ MNS } \\
\hline$(1388,4024,7652)$ & $0.8 \mathrm{~s}$ & $<0.1 \mathrm{~s}$ \\
$(2691,5998,-6067,12368)$ & & $1 \mathrm{MNS}$ \\
& $2.8 \mathrm{~s}$ & $0.1 \mathrm{~s}$ \\
$(1585,7818,-2542,-2803,5430)$ & $163.0 \mathrm{~s}$ & $1 \mathrm{MNS}$ \\
& & $1.4 \mathrm{~s}$ \\
$(479,7114,1909,-5696,192,18594)$ & $>5,400 \mathrm{~s}$ & $65.3 \mathrm{~s}$ \\
$(1038,22,-2)$ & $>900 \mathrm{Mo}$ & $8 \mathrm{MNS}$ \\
$(1021,37,-40,178)$ & $0.8 \mathrm{~s}$ & $0.1 \mathrm{~s}$ \\
& $12.2 \mathrm{~s}$ & $3 \mathrm{MNS}$ \\
$(1051,-45,26,-5,-131)$ & $195.4 \mathrm{~s}$ & $4 \mathrm{MNS}$ \\
$(1024,6,60,-6,-42,52)$ & $>10,800 \mathrm{~s}$ & $2.8 \mathrm{~s}$ \\
& $>2,000 \mathrm{Mo}$ & $42 \mathrm{MNS}$ \\
& & \\
\end{tabular}


Table 5. Computation time for LattE and our programs, for $D_{n}$.

\begin{tabular}{crr}
\hline Root lattice element & LattE & \multicolumn{1}{c}{ MNS } \\
\hline$(8608,-305,183)$ & $0.3 \mathrm{~s}$ & $\begin{array}{r}<0.1 \mathrm{~s} \\
1 \mathrm{MNS} \\
\end{array}$ \\
$(32,5914,6166,-5360)$ & $1.5 \mathrm{~s}$ & $<0.1 \mathrm{~s}$ \\
& $18.0 \mathrm{~s}$ & $0.5 \mathrm{~s}$ \\
$(1646,3916,-3330,6372,7452)$ & $313.5 \mathrm{~s}$ & $2 \mathrm{MNS}$ \\
& & $3.1 \mathrm{~s}$ \\
$(8127,601,-2870,-2908,10823,3639)$ & $0.2 \mathrm{~s}$ & $<0.1 \mathrm{~s}$ \\
$(1009,1106,-9)$ & $3.0 \mathrm{~s}$ & $1 \mathrm{MNS}$ \\
$(1074,959,64,77)$ & & $6.3 \mathrm{~s}$ \\
& $100.2 \mathrm{~s}$ & $3.1 \mathrm{~s}$ \\
$(1100,973,2,-1,-60)$ & & $18 \mathrm{MNS}$ \\
$(1096,965,-54,68,-34,-1)$ & $7,076.7 \mathrm{~s}$ & $763.3 \mathrm{~s}$ \\
& & $47 \mathrm{MNS}$ \\
\hline
\end{tabular}

Tests were performed on Pentium IV 1 and $13 \mathrm{GHz}$ computers with 1500 or 2000 mega-octets (Mo) of RAM memory. We stopped several computations with LattE when we figured out that they would overcome the computers' memory or take too much time with respect to the other algorithms; in this case we indicate the time spent and the number of mega-octets used by the computer.

\section{Acknowledgments}

During the course of this work, we have benefited from discussions with Jesús de Loera, András Szenes, Corrado de Concini, Claudio Procesi, and an anonymous referee. We thank them for sharing their mathematical expertise with us. We also thank the various institutions that helped us to collaborate on this work: the Research-in-pairs program at the Forschungsinstitut Oberwolfach, a LIEGRITS grant and the University Tor Vergata Roma, the University Denis Diderot in Paris, the Centre Laurent Schwartz at Ecole Polytechnique, and San Francisco State University.

\section{References}

1. Baldoni-Silva, W., and Vergne, M., Residues formulae for volumes and Ehrhart polynomials of convex polytopes (2001), available at math.ArXiv, CO/0103097.

2. Baldoni-Silva, W., De Loera, J.A., and Vergne, M., Counting integer flows in networks, Found. Comput. Math. 4 (2004), 277-314, available at math.ArXiv, CO/0303228.

3. Barvinok, A.I., A polynomial time algorithm for counting integral points in polyhedra when the dimension is fixed, Math. Oper. Res. 19 (1994), 769-779.

4. Beck, M., and Pixton, D., The Ehrhart polynomial of the Birkhoff polytope, Discrete Comput. Geom. 30(4) (2003), 623-637.

5. Beck, M., and Zaslavsky, T., The number of nowhere-zero flows on graphs and signed graphs (2004), available at math.ArXiv, CO/0309331. 
6. Beyls, K., Bruynooghe, M., Loechner, V., Seghir, R., and Verdoolaege, S., Analytical computation of Ehrhart polynomial and its applications for embedded systems, IEEE/ACM International Symposium on Code Generation, Palo Alto, California, March 2004.

7. Brion, M., and Vergne, M., Residue formulae, vector partition functions and lattice points in rational polytopes, J. Amer. Math Soc. 10(4) (1997), 797-833.

8. Brion, M., and Vergne, M., Arrangements of hyperplanes, I: Rational functions and Jeffrey-Kirwan residue, Ann. Sci. École Norm. Sup. 32 (1999), 715-741.

9. Cochet, C., Multiplicities and tensor product coefficients for $A_{r}$ (2003), available at math.ArXiv, $\mathrm{CO} / 0306308$.

10. Cochet, C., Vector partition function and representation theory (2005), available at math.ArXiv, $\mathrm{RT} / 0506159$

11. homepage http://www.math.jussieu.fr/ $\sim_{\text {cochet/ }}$

12. Dahmen, W., and Micchelli, C.A., The number of solutions to linear diophantine equations and multivariate splines, Trans. Amer. Math. Soc. 308 (1988), 509-532.

13. De Concini, C., and Procesi, C., Nested sets and Jeffrey-Kirwan cycles (2004), available at math.ArXiv, $\mathrm{AG} / 0406290$.

14. De Loera, J.A., Haws, D., Hemmecke, R., Huggins, P., Tauzer, J., and Yoshida, R., A User's Guide for LattE v1.1 (2003), software package LattE, available at http://www. math.ucdavis.edu/ ratte/

15. De Loera, J.A., Hemmecke, R., Tauzer, J., and Yoshida, R., Effective lattice point enumeration in rational convex polytopes, J. Symbolic Comput. 38(4) (2004), 1273-1302.

16. Ehrhart, E., Polynômes arithmétiques et méthode des polyèdres en combinatoire, IRMA, Strasbourg (1975).

17. Jeffrey, L.C., and Kirwan, F.C., Localization for nonabelian group actions, Topology 34 (1995), 291-327.

18. Khovanskii, G., and Pukhlikov, A.V., A Riemann-Roch theorem for integrals and sums of quasipolynomials over virtual polytopes, St. Petersburg Math. J. 4 (1993), 789-812.

19. Lee, J., Subspaces with well-scaled frames, Linear Algebra Appl. 114/115 (1989), 21-56.

20. Sturmfels, B., On vector partition functions, J. Combin. Theory Ser. A 72(2) (1995), 302-309.

21. Szenes, A., Iterated residues and Bernoulli polynomials, Int. Math. Res. Not. 18 (1998), 937-956.

22. Szenes, A., and Vergne, M., Residue formulae for vector partitions and Euler-MacLaurin sums, $A d v$. in Appl. Math. 30 (2003), 295-342.

23. Szenes, A., and Vergne, M., Toric reduction and a conjecture of Batyrev-Materov, Inv. Math. 158 (2004), 453-495.

24. Vergne, M., Residue formulae for Verlinde sums, and for number of integral points in convex rational polytopes, Proceedings of the Tenth General Meeting of the European Women in Mathematics, Malta, August 2001, pp. 223-285, World Scientific, River Edge, NJ (2003).

Received May 6, 2005, and in revised form November 13, 2005. Online publication April 28, 2006. 